\title{
VERA Transient Capability to Support ATF/High Burnup Fuel/HALEU Conversion
}

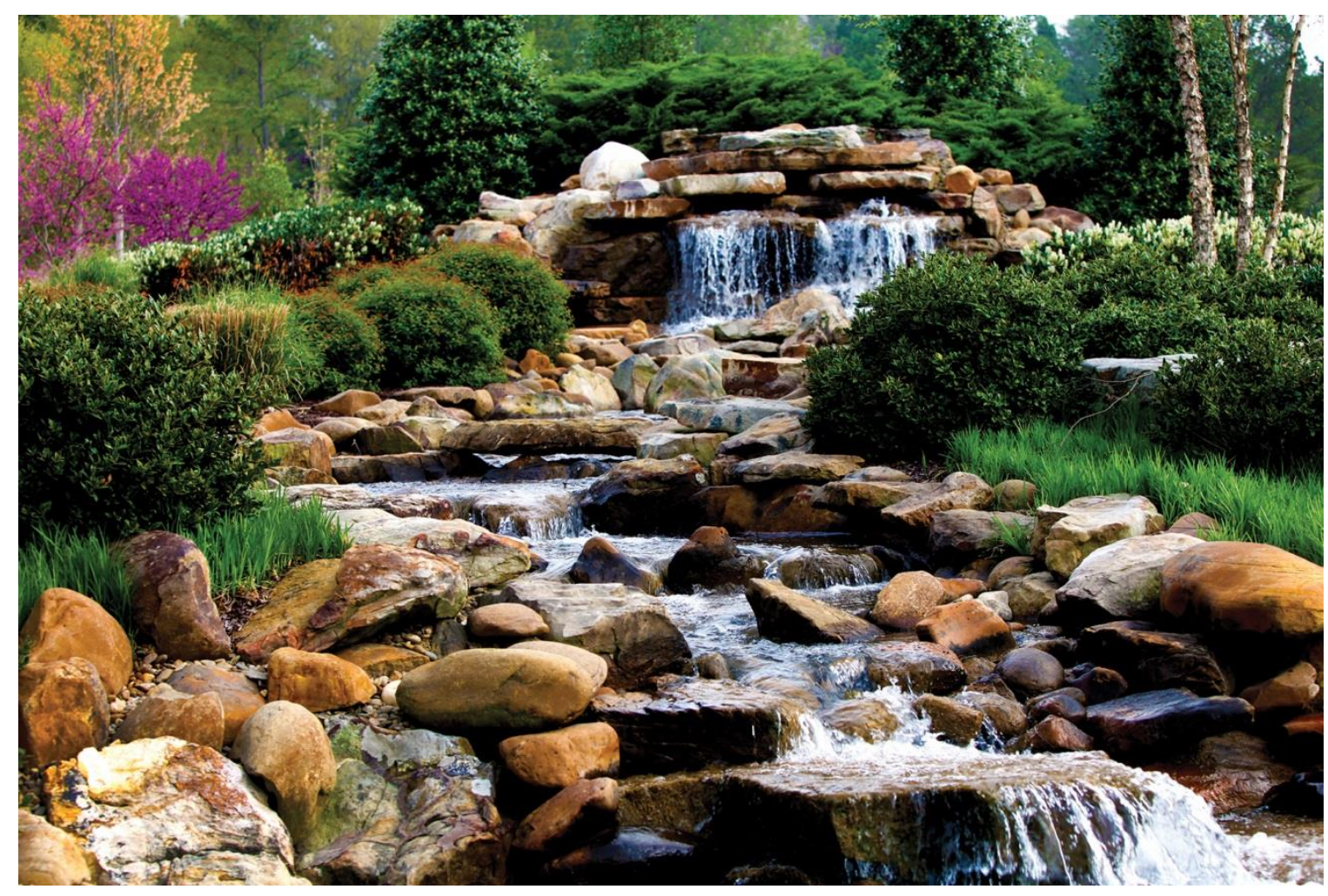

Aaron Graham

Shane Henderson

Bob Salko

Aaron Wysocki

Ben Collins

November 2021

National Laboratory 


\title{
DOCUMENT AVAILABILITY
}

Reports produced after January 1, 1996, are generally available free via US Department of Energy (DOE) SciTech Connect.

Website www.osti.gov

Reports produced before January 1, 1996, may be purchased by members of the public from the following source:

\author{
National Technical Information Service \\ 5285 Port Royal Road \\ Springfield, VA 22161 \\ Telephone 703-605-6000 (1-800-553-6847) \\ TDD 703-487-4639 \\ Fax 703-605-6900 \\ E-mail info@ntis.gov \\ Website http://classic.ntis.gov/
}

Reports are available to DOE employees, DOE contractors, Energy Technology Data Exchange representatives, and International Nuclear Information System representatives from the following source:

Office of Scientific and Technical Information

PO Box 62

Oak Ridge, TN 37831

Telephone 865-576-8401

Fax 865-576-5728

E-mail reports@osti.gov

Website https://www.osti.gov/

This report was prepared as an account of work sponsored by an agency of the United States Government. Neither the United States Government nor any agency thereof, nor any of their employees, makes any warranty, express or implied, or assumes any legal liability or responsibility for the accuracy, completeness, or usefulness of any information, apparatus, product, or process disclosed, or represents that its use would not infringe privately owned rights. Reference herein to any specific commercial product, process, or service by trade name, trademark, manufacturer, or otherwise, does not necessarily constitute or imply its endorsement, recommendation, or favoring by the United States Government or any agency thereof. The views and opinions of authors expressed herein do not necessarily state or reflect those of the United States Government or any agency thereof. 
ORNL/SPR-2021/2325

Nuclear Energy Advanced Modeling and Simulation (NEAMS) Program

\title{
VERA TRANSIENT CAPABILITY TO SUPPORT ATF/HIGH BURNUP FUEL/HALEU CONVERSION
}

\author{
Aaron Graham, Shane Henderson, Bob Salko, Aaron Wysocki, Ben Collins
}

November 2021

Prepared by OAK RIDGE NATIONAL LABORATORY

Oak Ridge, TN 37831-6283

managed by

UT-BATTELLE LLC

for the

US DEPARTMENT OF ENERGY

under contract DE-AC05-00OR22725 



\section{CONTENTS}

\section{Contents}

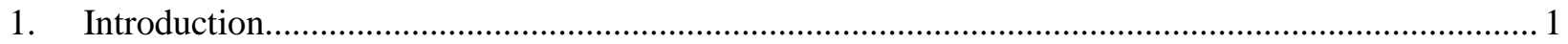

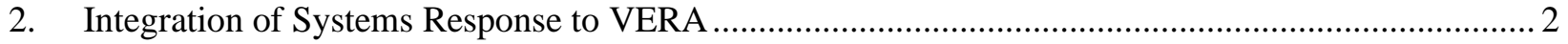

3. Implementation of Transient Checkpoint Capability ................................................................... 2

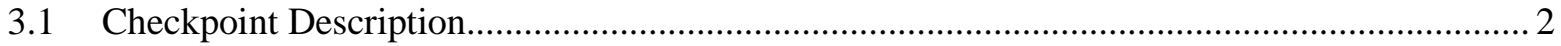

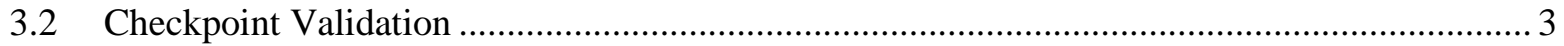

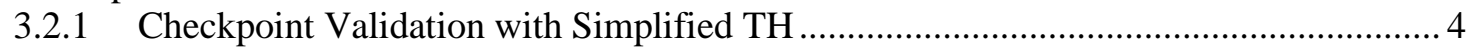

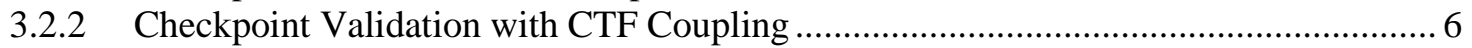

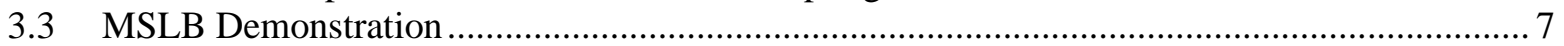

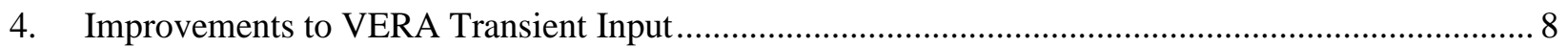

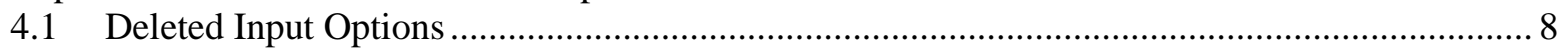

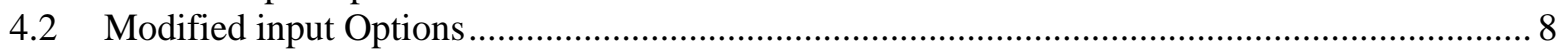

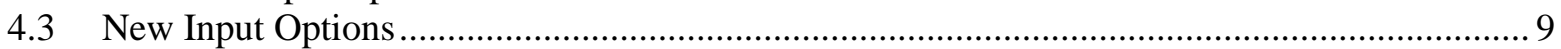

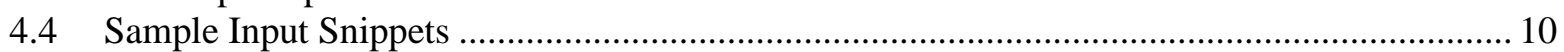

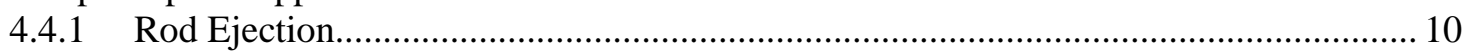

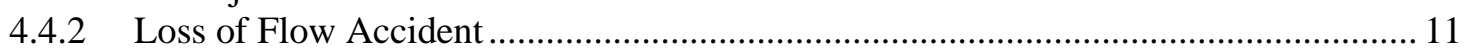

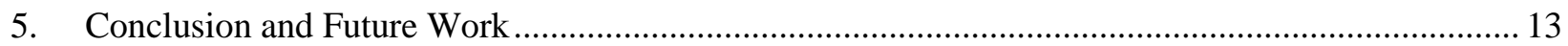

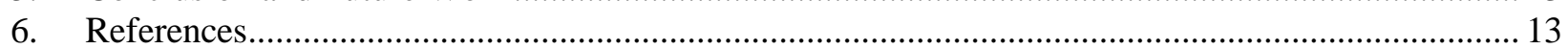

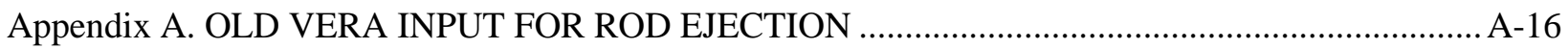

Appendix B. OLD VERA INPUT FOR LOSS OF FLOW ........................................................... B-29

Appendix C. NEW VERA INPUT FOR EXACT LOSS OF FLOW …........................................... C-33 


\section{INTRODUCTION}

The Virtual Environment for Reactor Applications (VERA) [1] was developed under the Consortium for Advanced Simulation of Light Water Reactors (CASL). The goal of this new code suite was to provide high-fidelity, whole-core simulation using pin-resolved physics. Extensive steady-state validation of VERA has been performed for reactor cycle depletion simulations and other related simulations [2,3,4,5]. Transient simulations were not a major component of the CASL work; reactivity insertion accidents (RIAs) received most of the focus [6,7]. However, there are other design basis accidents (DBA) which are of great importance for reactor design and licensing.

Some of these accidents, such as a main steam line break (MSLB) or other similar loss of flow (LOF) accidents are much slower evolving than a RIA. Much of the physics of interest in a RIA take place within a few seconds because the accident is largely neutronics-driven. However, the thermal hydraulic (TH) effects, including the primary loop outside the reactor core (and its interaction with the secondary loop for PWRs), is of much greater importance for LOF accidents. This means that the transients take several minutes to fully evolve, as opposed to a few seconds. This milestone report documents improvements made to VERA to enable modeling of a wider variety of transients that are important to the development of accident-tolerant and high burnup fuels.

There were two major requirements for VERA to be able to model slowly evolving transients described above. First, systems-level feedback (primary loop outside the core, secondary loop and heat exchanger, pump) is required to obtain correct coolant conditions at the core inlet and correct system pressure over time. VERA did not initially include any systems coupling, but this issue was addressed in NEAMS Milestone M3MS-21OR0701045 "Integration of Systems Response to VERA," completed in June 2021 and summarized in Section 2 of this report.

The other major requirement was to address the long runtime of such a calculation. Although VERA provides high-fidelity solutions that are significantly more detailed and accurate than many legacy methods, this fidelity comes with significantly increased computational expense. Many high-performance computing (HPC) platforms have a maximum walltime for a single calculation. For reactor cycle depletions and fast-running transients this is not an issue, but it is for long-running transients such as a MSLB or LOF. To ensure that these calculations can be completed, it was necessary to implement a checkpoint system in VERA. This checkpoint system can write all the current solver data to a binary file at any point in the transient simulation, then resume the calculation by reading that file. This allows the transient calculation to be split up into multiple job submissions on an HPC platform, with each individual submission running for less than the maximum walltime. Section 3 of this report provides additional details on the functionality of the checkpoint system as well as some results showing its use for transient calculations.

The final improvement made to VERA as part of this milestone was to simplify the input. As mentioned previously, the focus of VERA development under CASL was on steady-state simulation with some effort toward RIA simulations. As such, the inputs that were added to describe transient simulations were specific to the needs at that time and not easily extended to other types of transients. To resolve this, an extension to VERAIn [8] was developed that includes a new input block for transient calculations. These updates greatly improve the flexibility of VERA for transient calculations and make it far less error prone. This is discussed in Section 4 of the report. 


\section{INTEGRATION OF SYSTEMS RESPONSE TO VERA}

In previous work, a preliminary coupling was set up between CTF [9] and the US Nuclear Regulatory Commission TRAC/RELAP Advanced Computational Engine (TRACE) system code [10]. The Extended Coupling Interface (ECI) offered by TRACE was used to perform the coupling. In a more recent NEAMS milestone, documented in Wysocki et al. [11], this coupling was improved by implementing a domainoverlapping coupling approach, in which TRACE solves the core region as a coarse, 1D component, so that the entire flow loop is still solved by TRACE. The core inlet boundary conditions are still passed to CTF and CTF will set the core pressure drop in TRACE by adjusting a valve opening. In this way, a twoway coupling is achieved; however, the approach has been shown to improve the numerical stability and performance of the initial capability that was developed in 2019.

In addition to the improvement of the coupling, an initial restart capability was implemented into CTF for modeling of long-running transients and support was added for running parallel CTF models when coupled to TRACE. A MSLB transient was run using the coupled CTF and TRACE capability to demonstrate the approach. It is noted that a nodal meshing approach was used in CTF to limit the number of processors needed in the solution of the full core geometry. This was done because it had been found that the runtime of the solution increases substantially when the number of processors used in the coupling increases beyond a certain threshold.

It was found that simply initializing the ECI (and not calling any other TRACE coupling procedures) leads to the slowdown for certain core configurations. Several pin-resolved cases were run on the Sawtooth HPC system to test this issue. For these cases, one processor was used for each assembly in the CTF core model, meaning that as problem size grows, the number of processors per assemblies remains constant. Note that only CTF is running in parallel when more than one processor is used. Whether CTF is running in serial or parallel, the TRACE portion of the simulation is performed on the single root process only and is therefore running in serial. All communication with TRACE is performed through the single root process of CTF. All CTF data that must be communicated to TRACE is first reduced to the CTF root process, then sent to TRACE; for TRACE data communicated to CTF, the data are sent to the CTF root process then broadcast to the other processes. The largest case that was able to be run was found to be 35 assemblies/processors. A 44-assembly case resulted in the ECI not being able to communicate between TRACE and CTF. A 48-assembly case was able to communicate but resulted in a severe increase in runtime. Although the 35 -assembly case was able to complete in 375 seconds, the 48assembly case did not finish in the 3600 job walltime limit. Note that all of these core configurations use only one node of the machine and similar behavior has been observed on a different HPC system.

Because of this, the coupled simulation is currently limited to smaller core configurations, coarse meshing strategies, or smaller pin-resolved problems.

\section{IMPLEMENTATION OF TRANSIENT CHECKPOINT CAPABILITY}

\subsection{CHECKPOINT DESCRIPTION}

The goal of the checkpoint file is to allow long running simulations to save their in-progress calculations and pick up where they left off in a second run. Fast transients such as RIAs often do not need this capability, but slower transients such as MSLBs are often intractable to run for long enough in a single calculation when using high-fidelity core simulators such as VERA.

To accomplish this, extensive modifications were made to MPACT [12,13] — which acts not only as the neutronics solver but also as the multiphysics driver for many VERA calculations - to allow it to generate a checkpoint file. The goal of this checkpoint file is for each of the solvers to write all their current and 
previous timestep solution data to an HDF5 file; this is done so that in a second calculation, reading this file is sufficient to resume the calculation without repeating any portion of the steady-state solve or the previously completed part of the transient solve.

One major limitation that was decided at the outset was to require that the user use exactly the same input file when resuming the transient calculation, except for changing the checkpoint_write input to a checkpoint_read input. This requirement should not be a burden on the user because realistic calculations generally do not try to change geometry, mesh, materials, or solver options in the middle of a transient calculation, but it greatly simplifies the size of the checkpoint file and the ease of implementing the capability. This requirement implies that the user must also use the same parallel decomposition when reading the checkpoint. Eventually some pieces of this requirement may be relaxed, but for now the benefits greatly outweigh the drawbacks.

The data written to the checkpoint files includes the following:

- Region-wise and group-wise scalar fluxes for the current and previous timestep,

- Region-wise and group-wise adjoint fluxes,

- Region-wise temperatures and densities,

- State date such as power, flow, pressure, etc., and

- Various internal solver data that is necessary to aid the calculations.

Much other data, such as geometry and mesh data and isotopic distributions, do not need to be written to the checkpoint file because of the requirement described in the previous paragraph. All these data can be reconstructed exactly during initialization using information in the input file. This allows the code to assume that the data in the checkpoint file is of the correct shape, removing any need for reshaping and mapping data after reading the checkpoint file.

A similar restart capability was implemented in CTF as documented in Wysocki et al. [11]. As noted in that document, there were small differences between the full transient and restarted transient test cases that were run when using the initial capability. As part of this work, this feature was further developed by finding additional solution parameters that must be included in the checkpoint dataset. After including the additional data, it was demonstrated that the solution is now exactly the same between the full transient and restarted transient for the restart test cases included in the CTF test matrix. These changes were also found to be necessary to keep the coupled MPACT/CTF solution consistent between the full and restarted transient.

\subsection{CHECKPOINT VALIDATION}

To ensure that the new checkpoint capability is working correctly, a small test problem was used. The problem is based on progression problem 4 of the VERA benchmark problems [2], which is a $3 \times 3$ assembly problem with a control rod inserted in the center assembly. Three primary changes are made to this problem to obtain what is termed the 4-mini problem:

1. The assemblies are changed from $17 \times 17$ pins to $7 \times 7$ pins, and their height is reduced from 12 feet to about 8 feet;

2. TH coupling is added;

3. The problem is converted to a transient problem; the control rod is withdrawn from 0 steps to 40 steps (out of 230 steps total) in 0.025 seconds, followed by 0.005 seconds of no additional movement.

Although this is a simplified problem, it requires all the same code mechanics as a full-scale transient but can easily be run on small development clusters instead of requiring HPC platforms. Thus, it serves as a useful initial demonstration of the checkpoint capability. 
Since the checkpoint capability is not aimed at adding any new physics, the only requirement for validating it is that VERA generates the same solution when using the checkpoint file as when running the entire transient in a single calculation. Thus, it is sufficient to simply run the full transient, and then run the transient again in two segments using the checkpoint file and compare the results to ensure sufficient accuracy.

\subsubsection{Checkpoint Validation with Simplified TH}

First, the 4-mini test problem was run using MPACT's simplified TH. This calculation is not as high fidelity as CTF and neglects many effects, but it gives a good approximation of the TH feedback effects. Additionally, it removes one possible source of discrepancy between the checkpoint and regular calculations by limiting the entire calculation to MPACT instead of other components of VERA.

Initially, the checkpoint was set to occur immediately after the steady-state solve. This can be seen at the end of the first portion of the calculation below.

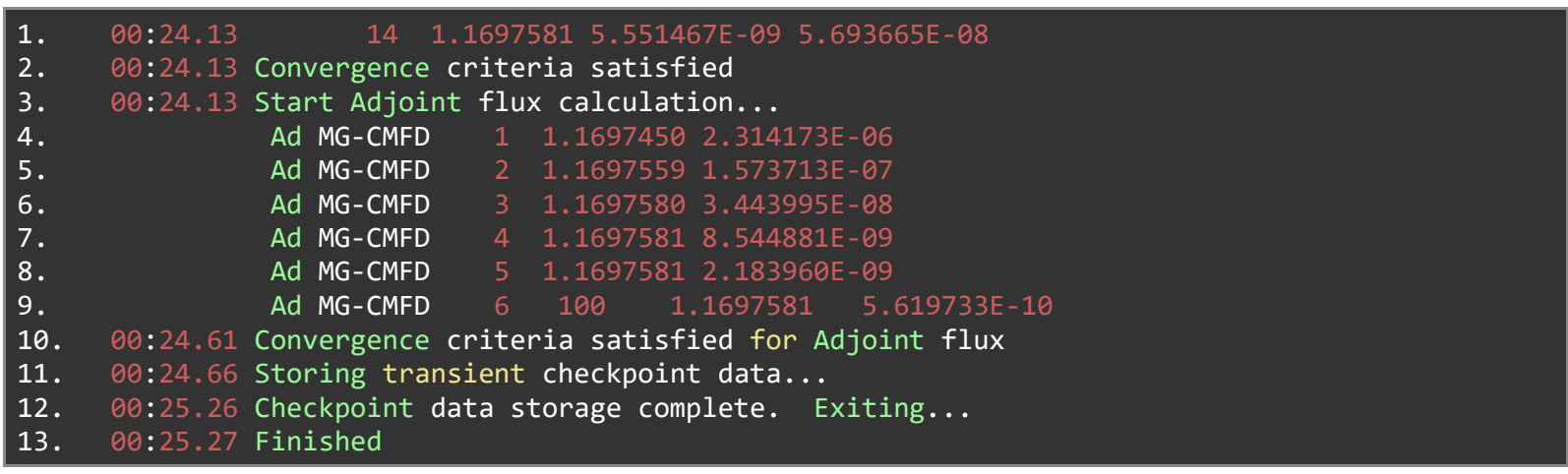

Upon reading in the checkpoint, the following is shown after processing input and initializing each of the solver objects.

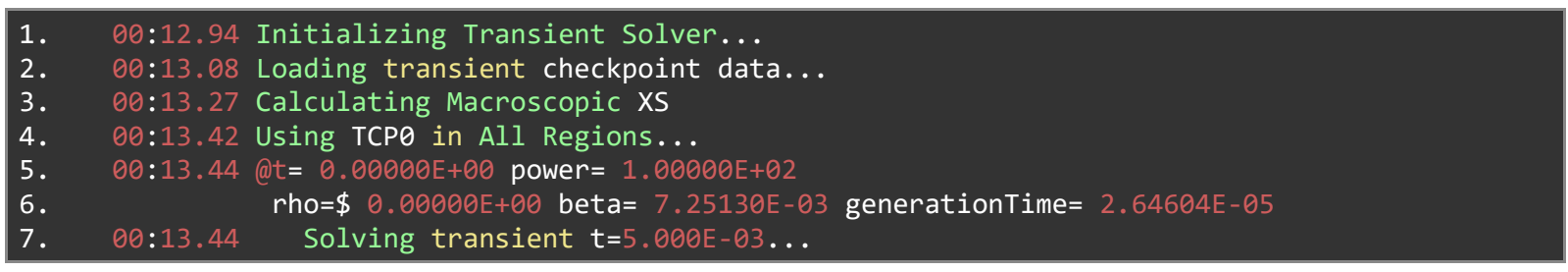

It can be seen in the second printout that the checkpoint file is loaded, a summary of the last timestep before the checkpoint is displayed (in this case, the steady-state solve at $\mathrm{t}=0.0$ ), and then a message is given showing that the calculation is resuming at the next transient timestep.

MPACT has a utility program called MPACTdiff.exe that is used for comparing code solutions to reference files as part of its automated testing script. Applying this utility to the results of the 4-mini transient calculation results allows for confirming the correctness of the checkpoint file. The full transient run was used as the reference case, and the checkpointed version associated with the above messages was used as the test case. The results for selected datasets on the final timestep are shown below.

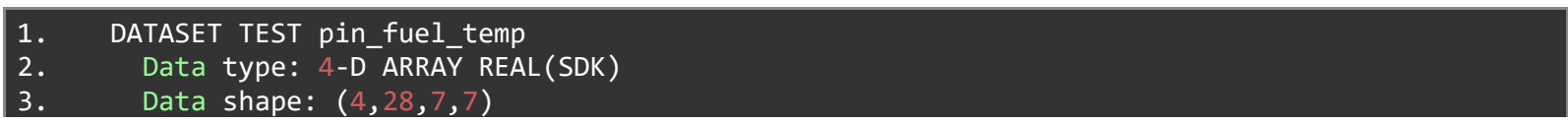




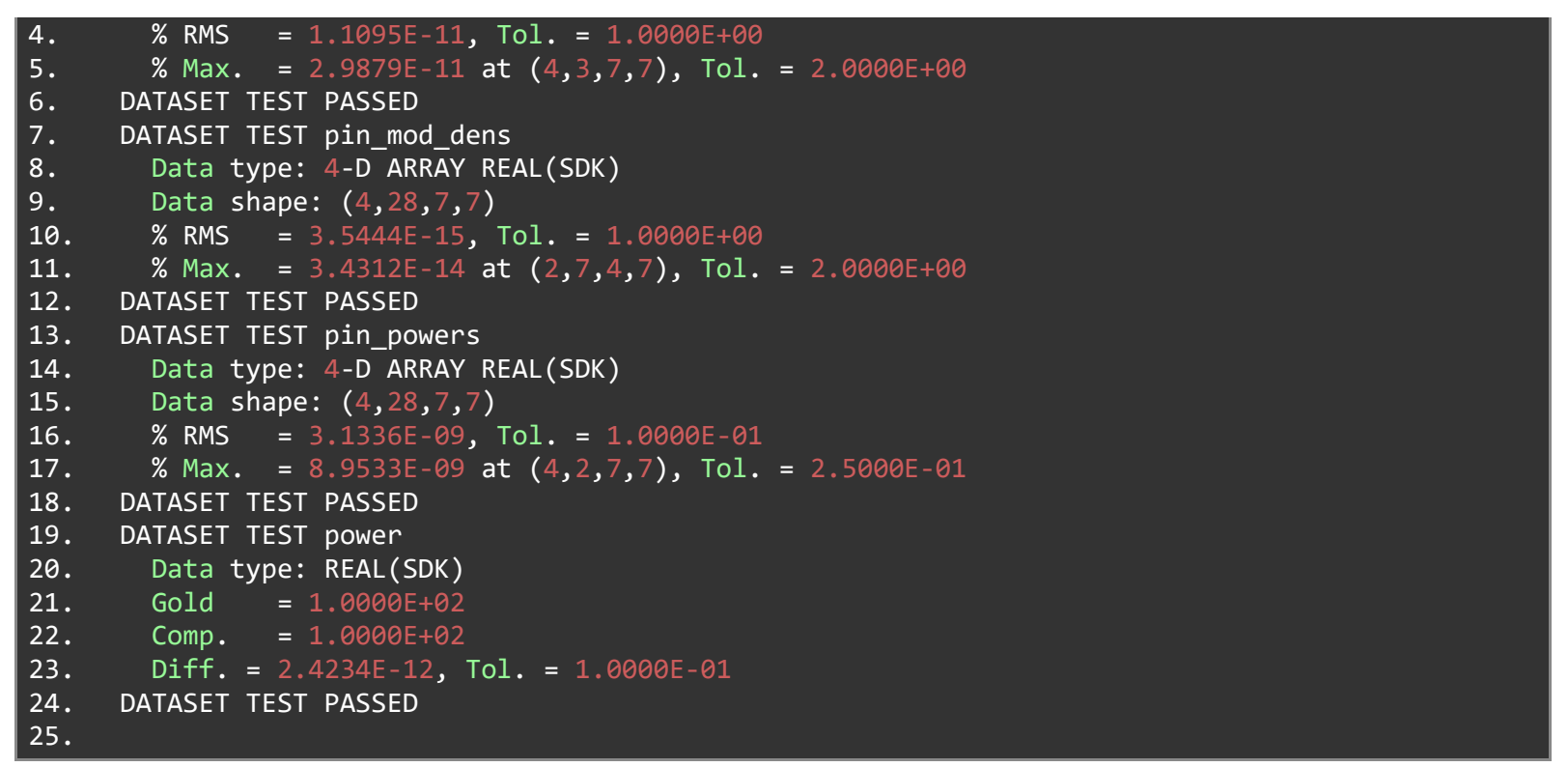

The maximum difference in 3D pin powers was less than $0.00000001 \%$ and the maximum difference in 3D fuel temperatures, 3D coolant densities, and total core power were all even smaller. Since this is the final timestep of the transient, these are the largest differences of any timestep. This shows that the checkpoint file worked very well for this case. It is not reasonable to expect closer agreement because of differences in the order of operations that contribute to round-off errors as well as mixed use of singleand double-precision variables.

An additional test performed using 4-mini and STH is to perform the same comparison again, changing the checkpoint to occur at $\mathrm{t}=0.025$ when the control rod reaches its maximum withdrawal position. This results in the following messages when writing the checkpoint file.

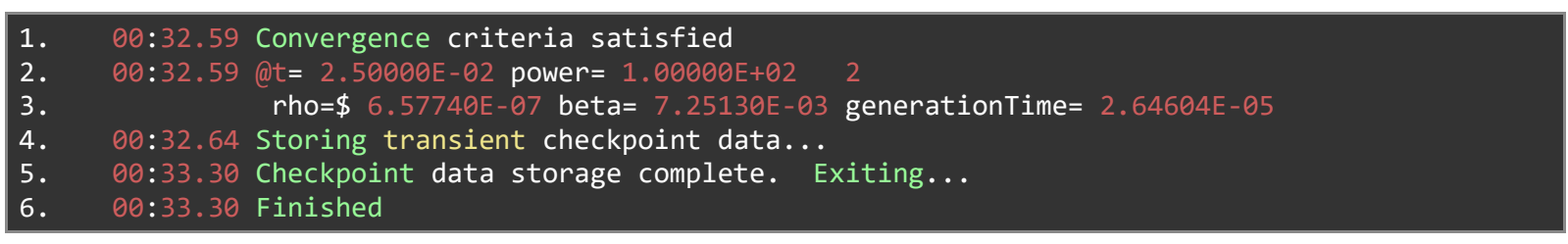

And the corresponding messages are shown upon reading the file, again showing that the calculation picks up at the proper time.

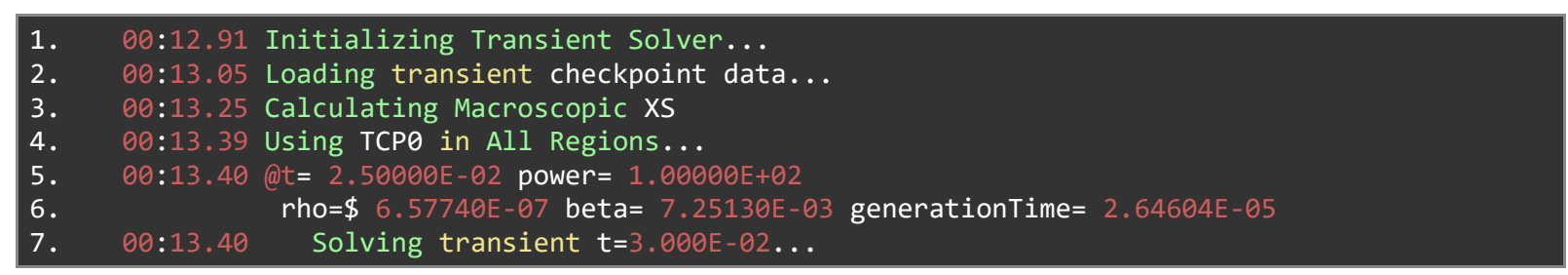

Running MPACTdiff.exe again using this version of the checkpoint file produces similar results to those produced the first time. The maximum pin power difference is even smaller than before because only a single timestep is taken after reading the checkpoint file, resulting in no drift of the solution. 


\subsubsection{Checkpoint Validation with CTF Coupling}

Since VERA calculations should ultimately be run using CTF coupling to obtain a sufficiently accurate TH solution, the two checkpoint tests in the previous section were repeated in the same manner, the only difference being that the simplified TH model was replaced with CTF coupling. For the first of the two tests, with the checkpoint occurring immediately after the steady-state solve, the following MPACTdiff.exe results are obtained.

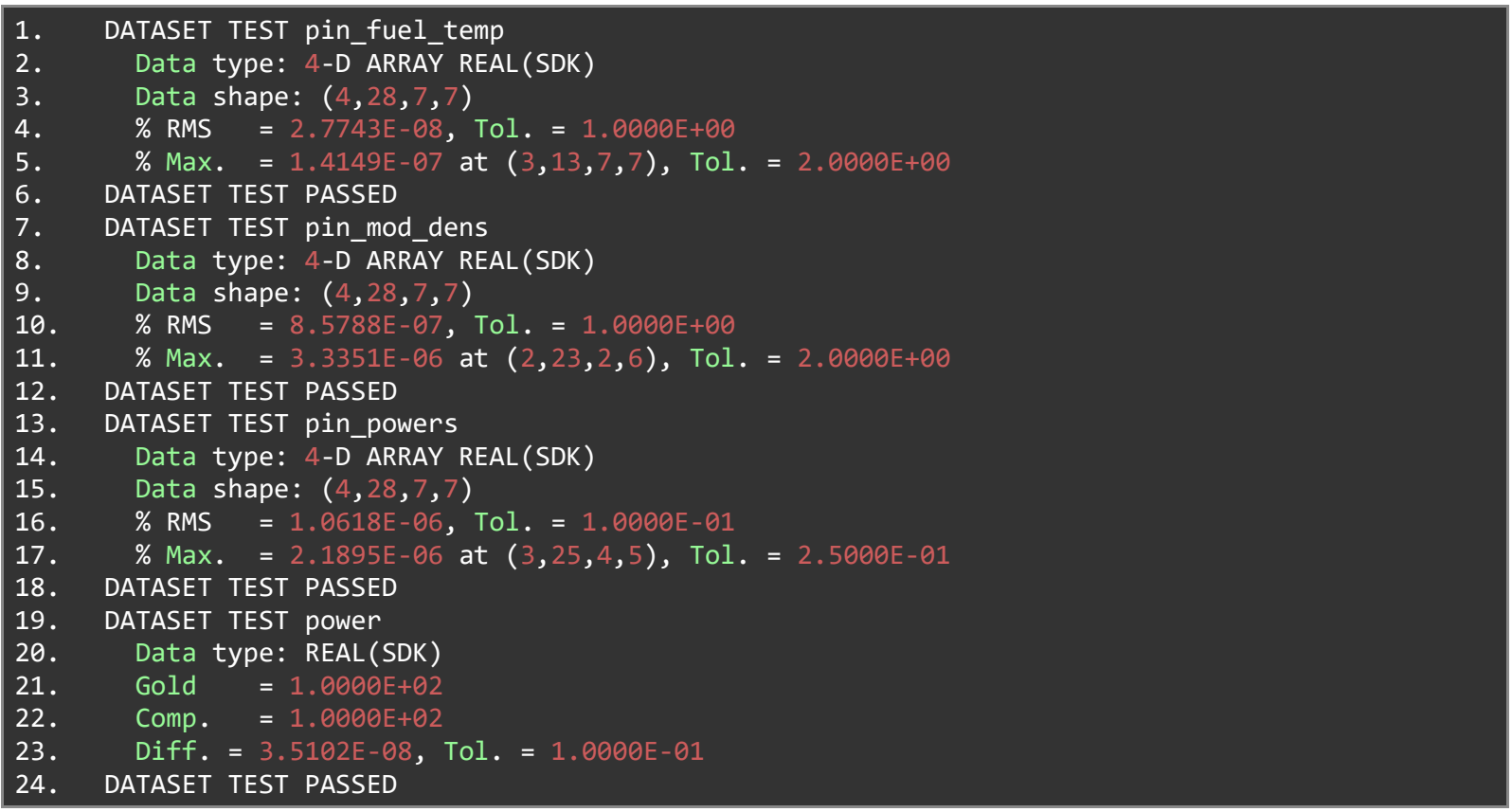

When performing the checkpoint at $\mathrm{t}=0.025$ seconds, the results below are obtained.

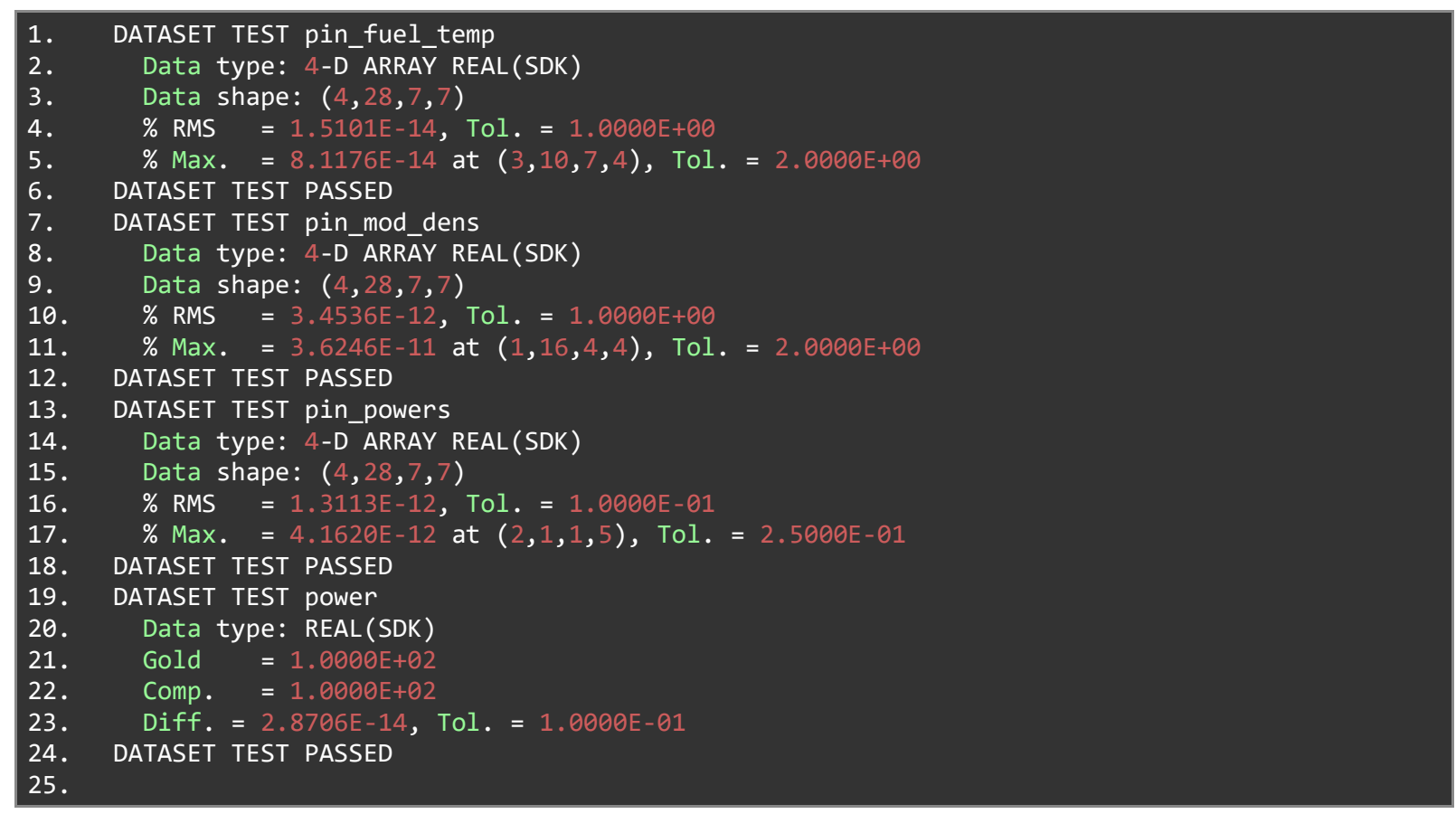


In both cases, the results are sufficiently close for practical applications. The differences are larger than those with simplified TH because the CTF calculation is significantly more complex. Many different models must be set up, requiring many datasets to be written to the checkpoint file compared only to a couple of datasets required for the simplified TH calculation. This allows more room for initialization to be done in a slightly different order for CTF when using the checkpoint file compared to without, causing some small solution drift. However, the amount of solution drift shown here is far smaller than what would impact the accuracy of full-scale calculations, so the current implementation of the checkpoint file system is deemed acceptable.

\subsection{MSLB DEMONSTRATION}

For a full-scale demonstration of the checkpoint capability, a miniature version of VERA progression problem 7 [2], referred to as 7-mini, was modified to contain a transient calculation. The transient used was a LOF accident. The transient portions of the input can be found in APPENDIX B, whereas the remainder of the input simply defines the geometry from Godfrey [2]. This provides a demonstration of the capability on exactly the type of problem it is intended for. The geometry and energy group changes to problem 7 to produce 7 -mini were the same as those to problem 4 to produce 4-mini. It was originally planned to use the full-sized problem 7, but system issues on HPC platforms prevented that. 7-mini was used as a surrogate because it uses all the same code features but can fit on smaller development clusters that did not have issues at the time these cases were run.

The calculation is $10 \mathrm{~s}$ of transient. This is shorter than the target application, but sufficient to demonstrate the checkpoint capability. It was modified to write a checkpoint file after the steady-state solve and $2 \mathrm{~s}$ of transient and then to read the checkpoint file back in for the remaining $8 \mathrm{~s}$ of calculation. This was done on 4 nodes with 128 compute cores on the ROSS development cluster at Oak Ridge national laboratory. As with the other demonstrations, a non-checkpoint version of the calculation was also run to validate the correctness of the checkpoint file.

For each calculation, the steady-state portion of the solve took about $13.5 \mathrm{~m}$ to converge. The transient portion of the calculation then took 1.25 hours for the first 2 seconds and about 4.25 hours for the remaining 8 seconds, which was read in from the checkpoint file. Running the calculation all at once without using the checkpoint was only about 6 minutes faster than the checkpointed version, showing that minimal time is spent writing the checkpoint and reinitializing from it.

The results compared well with running the full transient calculation in one attempt. The core power drifted about $0.01 \%$ in the 8 seconds after the checkpoint. The maximum difference in 3D power distribution was $0.007 \%$ and the maximum difference in 3D fuel temperature distribution was only $0.025 \%$. While the solution drift is non-zero it is small enough not to have any significant impact on analysis. Additional work may be possible to further reduce the differences when using the checkpoint file.

The file size for the 7-mini checkpoint was about 6 GB. For a full-scale checkpoint using VERA problem 7 , the total file size is estimated to be about 200 GB based on the increased size of the geometry and greater number of energy groups that must be stored. This is quite large, but any computing system on which a code like VERA will run should have no problem handling several files of this size. Since the file is only intended to persist long enough for the next stage of the transient to execute, there is no need for long term storage. 


\section{IMPROVEMENTS TO VERA TRANSIENT INPUT}

Because transient calculations were fairly limited in scope under the CASL program, only limited work was performed toward developing VERAIn options for transient calculations. The work that was done was generally focused on RIAs such as a rod ejection, for which it was relatively straightforward to provide a simplified description of the transient. Thus, the old input involved specifying one [STATE ] block for each timestep when the rod was moving and then providing a list of timesteps in which the [STATE ] block inputs did not change. Furthermore, because of the RIA focus, the transient-specific inputs were all implemented in the [MPACT] block of VERAIn, which is the portion of the input that provides code-specific options to MPACT, the neutron transport component of VERA.

The fact that the transient inputs were focused on RIA and MPACT has made it challenging to set up other types of transients, such as a MSLB or other LOF accidents. To address this challenge, a new [TRANSIENT] block was developed in VERAIn. This block focuses on providing high-level descriptions of the transient itself, without reference to specific codes, numerical methods, and so on. The new input seeks to keep the same concision as the rest of VERAIn while providing a high degree of flexibility.

It should be noted that although this is a significant overhaul of a portion of VERAIn, no change in functionality takes place in VERA. The new input simply improves the usability and reduces the likelihood of making input errors. It also should make it simpler to add new capabilities in the future compared to the old method of specifying a transient in the input.

The following sections will describe deleted input options, modified input options, and new input options, then provide some sample input snippets to demonstrate how transient specifications look in the old and new versions of VERAIn.

\subsection{DELETED INPUT OPTIONS}

The following input options were deleted from the [MPACT] block:

- perturb: this will be replaced by a new input option in the [TRANSIENT] block

- timestep: this will be replaced by a new timestep input in the [TRANSIENT] block

- prompt: this input caused an error if not provided, but had only one valid value

- accel: transient calculations are slow and should always use acceleration; acceleration is also required to determine the adjoint flux at the start of the transient, so this input only had one valid value

- $\quad$ summary_edits: summary edits will always be printed in some useful, concise way based on the edit_schedule input (see Section 4.3) instead of requiring the user to specify when to print these data

The following input option was deleted from the [STATE] block:

- bank_wd: this will be replaced by a new input option in the [TRANSIENT] block

\subsection{MODIFIED INPUT OPTIONS}

The following input options previously existed in the [STATE] block. However, they are not statedependent and are transient-specific; therefore, they have been moved to the new [TRANSIENT] block:

- scram_type

- scram_lock

- trip_time 
- trip_power

- trip_rate

\subsection{NEW INPUT OPTIONS}

The following input options will be added as part of the new [TRANSIENT] block:

- transient_time <end_time>

- Specifies how long the transient is run

- timestep $\left\langle d t \_1\right\rangle\left\langle t i m e \_1\right\rangle\left\langle d t \_2\right\rangle\left\langle t i m e \_2\right\rangle \ldots\left\langle d t \_n\right\rangle\left\langle t i m e \_n\right\rangle$

- Input is pairs of timestep size and the time until that timestep size is used

$\circ 0.0$ is implied as the starting time, so $\left\langle d t \_1\right\rangle$ is used between 0.0 and $\left\langle t i m e \_1\right\rangle$,

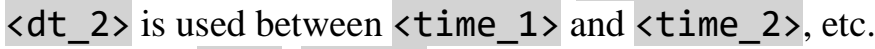

0 At least one $\langle d t\rangle$, $\langle$ time $\rangle$ pair must be specified

- The timesteps are effectively suggestions. Some components of VERA may have adaptive timestepping that would override this input. Additionally, timesteps will be split to align properly with physics perturbations, outputs, restart files, etc.

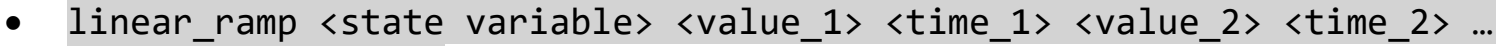
$\langle$ value_n〉 〈time_n〉

o The times must be in ascending order and must all be less than or equal to 〈end_time〉

0 If the final $\langle t i m e\rangle$ is less than $\langle$ end time $\rangle$, then the final $\langle v a l u e\rangle$ is used for the $\langle$ state variable $\rangle$ through the remainder of the calculation

- More than one linear_ramp input may be used to perturb multiple state variables together

- The variable will be linearly interpolated from the initial value to $\langle$ value_1 $\rangle$ between 0.0 and $\langle$ time_1 1$\rangle$, interpolated from $\langle$ value_1 $\rangle$ to $\langle$ value_2 $\rangle$ between $\langle$ time_1 $\rangle$ and $\langle$ time_2〉, etc.

- The state variables can be any of the following:

- power

- flow

- bypass

- tinlet

- subcool

- tfuel

- modden

- boron

- pressure

- kmul_beta

- kmul_doppler

- kmul_modtemp

- kmul_crw

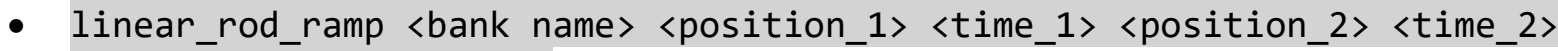

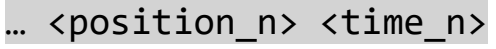

○ This input works the same as the linear_ramp input, but for moving control rods or blades

o <bank_name> should be a valid value from the bank_map input in the [CORE] block

- Linear interpolation will be performed on the rod positions using the same scheme as described in the linear_ramp input 
- linear_map_ramp $\langle$ map name $\rangle\left\langle m a p \_1\right\rangle\left\langle t i m e \_1\right\rangle\left\langle m a p \_2\right\rangle\left\langle t i m e \_2\right\rangle \ldots\left\langle m a p \_n\right\rangle$ 〈time_n>

- This input works the same as the linear_ramp input, but for inputs that require 2D assembly maps, such as blade_pos, void, tinlet_dist, pout_dist, flow_dist

- edit_schedule $\left\langle\mathrm{dt} \_1\right\rangle\left\langle\mathrm{time} \_1\right\rangle\left\langle\mathrm{dt} \_2\right\rangle\left\langle\mathrm{time} \_2\right\rangle \ldots\left\langle\mathrm{dt} \_\mathrm{n}\right\rangle\langle\mathrm{time} \mathrm{n}\rangle$

$\circ$ Specifies the timesteps to use for generating output edits; the meanings of the $\langle d t\rangle$ and <time> inputs are similar to previous inputs

- The calculation timesteps from the timestep input will be split to ensure that output edits are generated exactly at the specified timesteps

Additionally, the following new input will be added to the [STATE ] block:

- transient

O This should only appear in the first [STATE] block. Valid values are on or off.

- This is a convenience input. By putting transient off in the [STATE] block, a user can disable the transient portion of a calculation without removing the entire [TRANSIENT] block. If the [TRANSIENT] block is present and this input is not specified, then it defaults to transient on.

\subsection{SAMPLE INPUT SNIPPETS}

\subsubsection{Rod Ejection}

A recent project required the ejection of a control rod that was halfway inserted into the reactor. The ejection speed was assumed to be constant, with the rod fully exiting the top of the reactor in $1 \mathrm{~s}$. The reactor conditions were hot full power at the initiation of the accident. Because of the high reactivity insertion of the rod ejection, very small timesteps were required for VERA to remain stable. The old transient input requires one [STATE] block for every timestep in which something is changing. Thus, the rod ejection described here became very cumbersome, requiring 701 [STATE] blocks. The old version of the input is included in APPENDIX A. The new input, which is far more concise, is included below.

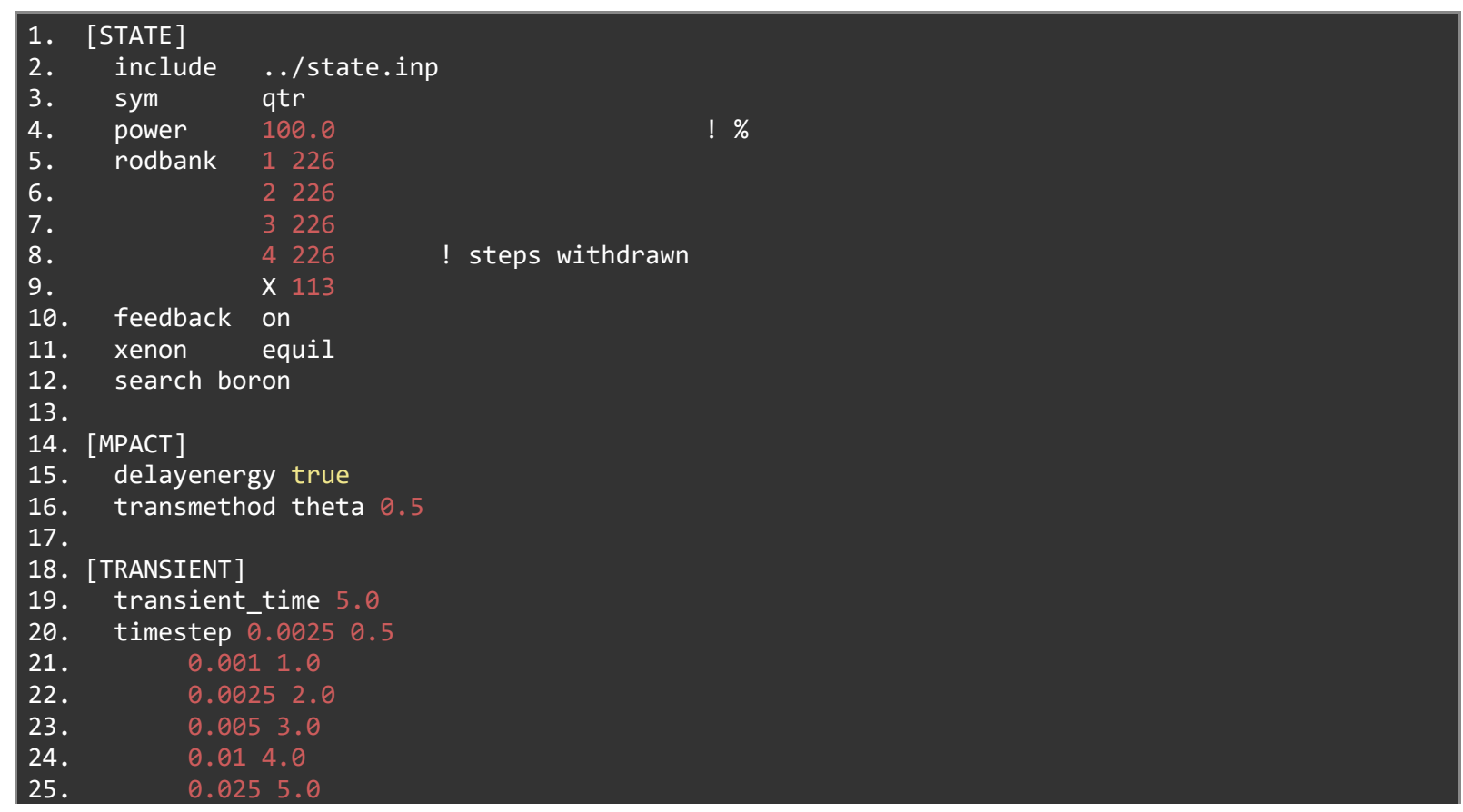




\subsubsection{Loss of Flow Accident}

A second example of the new input format is a LOF accident. These types of accidents typically require specifying outlet pressure and inlet flow rate over time. These functions are usually complicated, being the result of a separate systems code calculation. This transient demonstrates that the new input can be used either to replicate exactly the behavior of the old input, or dramatically simplify it through very minor approximations. The old input will be shown in APPENDIX B because of its length.

\subsubsection{LOF Exact New Input}

For this case, specifying the input exactly is still lengthy because of the complex shape of the pressure and flow functions required for the new input. There are still improvements in how the data are organized in the input, and the new input makes it easier for a user to copy and paste a table of time and state variable data. However, it does not significantly reduce the length of the input. For this reason, this version is shown in APPENDIX C.

\subsubsection{LOF Approximate New Input}

Significant simplification of the LOF input can be obtained by breaking the pressure and flow functions into piecewise linear functions. Figure 1 below shows the exact and piecewise linear forms of the flow and pressure. Visual inspection shows very little distinction between the two.

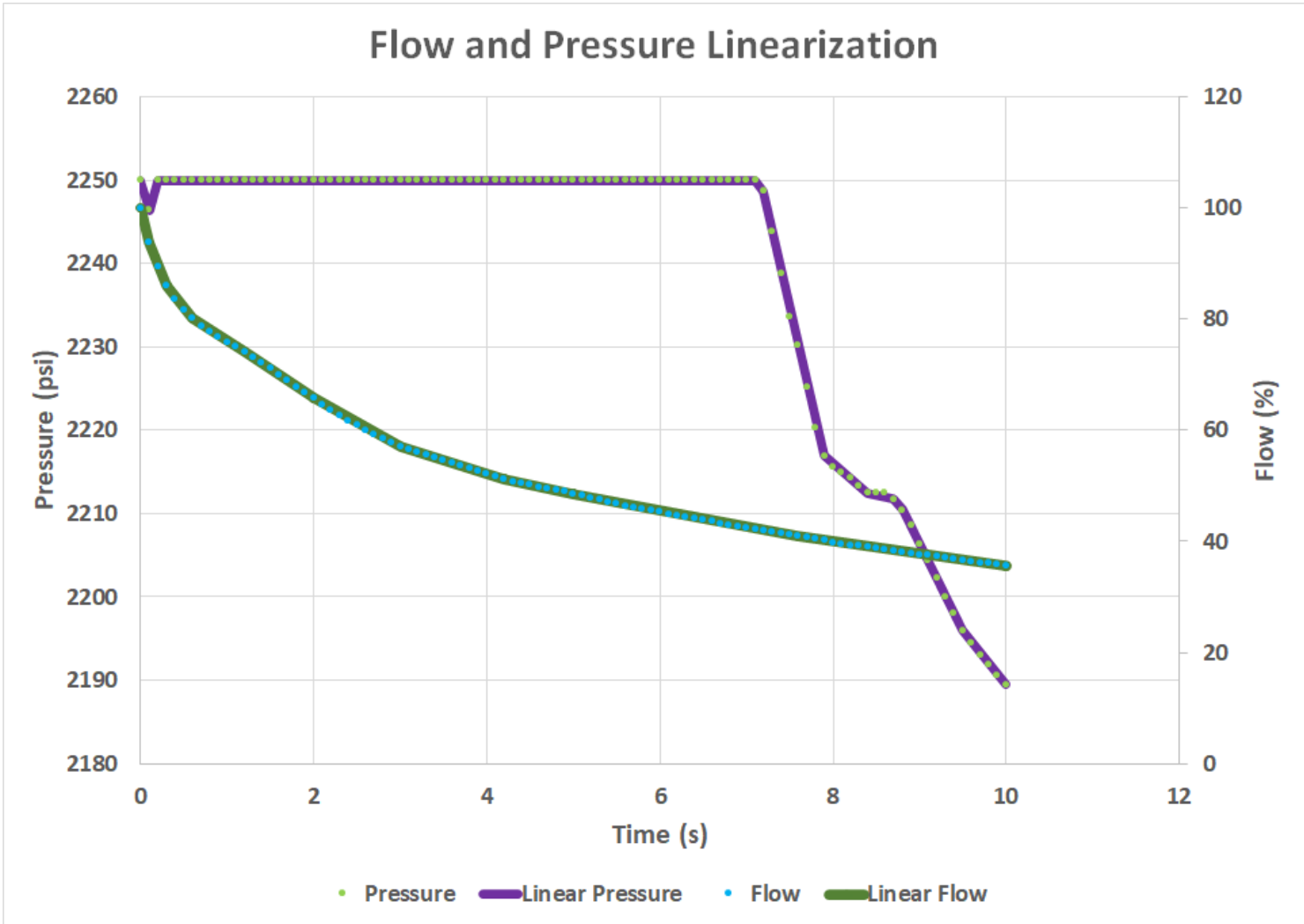

Figure 1. Comparisons of exact and piecewise linear versions of pressure and flow for LOF accident 


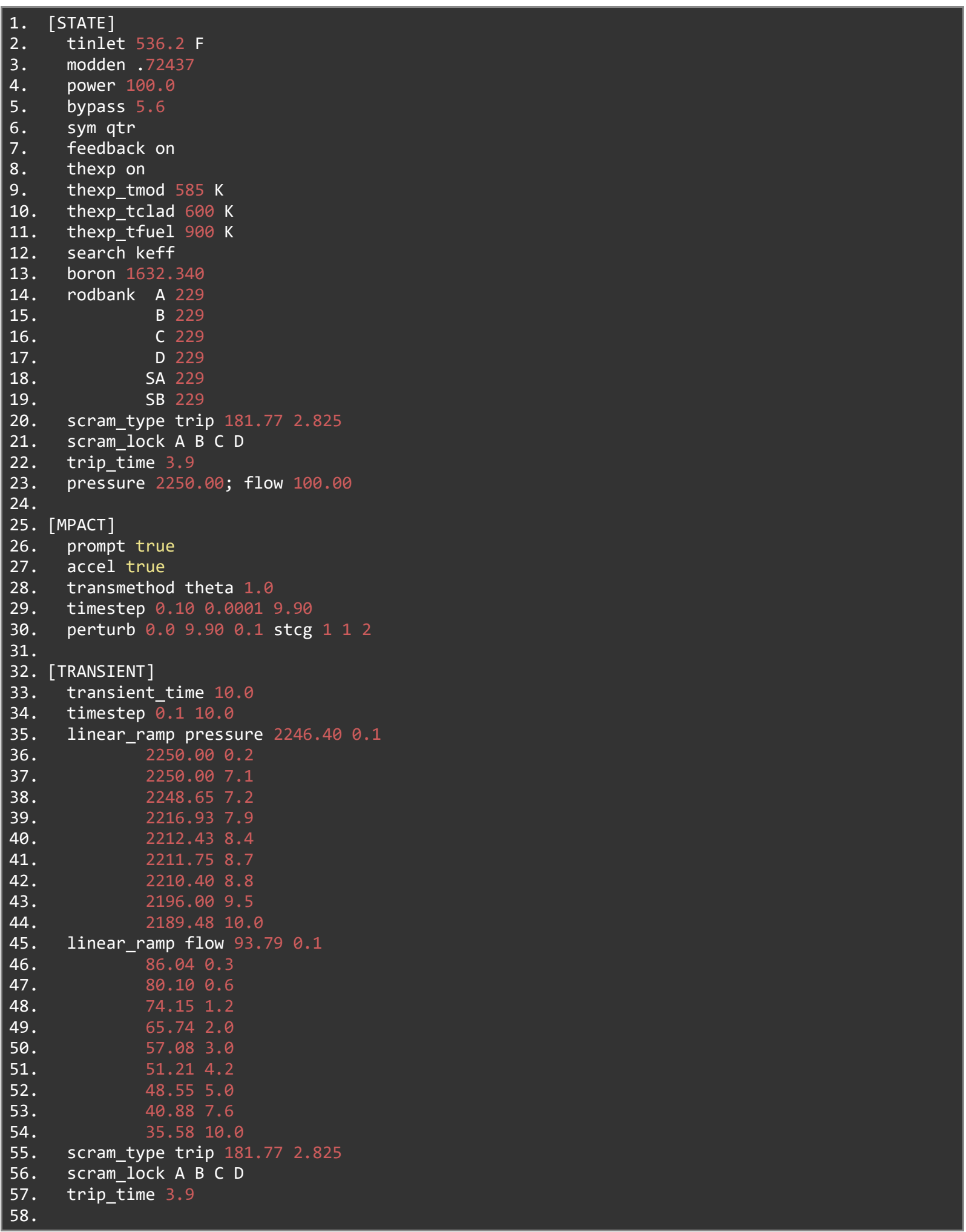




\section{CONCLUSION AND FUTURE WORK}

Several tasks were completed to improve VERA for long running transients necessary to analyze and qualify advanced fuels. First, a checkpoint file system was implemented as a means of dealing with VERA'S long runtimes. This allows the transient to be broken up into multiple pieces. Second, the transient input was updated to be much more user-friendly and flexible. Additionally, integration of the systems code TRACE with VERA was performed in a previous milestone; the work was summarized here because it is relevant to the calculations in this report.

The results with the checkpoint file demonstrate its effectiveness for a small test problem and a full-scale problem. This demonstration showed that the checkpoint file contains sufficient information to resume the calculation with no significant solution drift. For the TRACE coupling, issues were encountered when scaling up to large, parallel calculations. Simply having the code initialized resulted in major slowdowns, even if it was not called during the calculation. For this reason, the full-scale loss of flow calculation would be demonstrated only with the in-core VERA calculations.

Several items should be addressed moving forward. First, the TRACE issues must be resolved for it to be used for full-scale calculations. Otherwise, significant slowdowns will occur that will make it more challenging for users to run calculations with TRACE coupling enabled. Second, additional physics still need to be integrated into VERA. The primary example of this for long-running transients is decay heat. A significant portion of the reactor's heat is generated from decay heat, so even after the reactor shuts down, this heat is still being generated. For RIA calculations, this can be neglected, but for flow-related transients that run for several minutes, it is important to capture this. Finally, improving timestepping would make using VERA simpler. Currently, the user must provide timestep sizes for each portion of the calculation. It would be easier on the user if the timesteps could be dynamically calculated by the codes and modified based on the condition of the calculation.

\section{REFERENCES}

1. J. Turner, K. Clarno, M. Sieger, R. Bartlett, B. Collins, R. Pawlowski, R. Schmidt, and R. Summers, "The Virtual Environment for Reactor Applications (VERA): Design and Architecture," Journal of Computational Physics, 326, pp. 544-568 (2016), https://doi.org/10.1016/j.jcp.2016.09.003.

2. A. Godfrey, "VERA Core Physics Benchmark Progression Problem Specifications," Oak Ridge National Laboratory Technical Report CASL-U-2012-0131-004 (2014).

3. A. Godfrey, B. Collins, C. Gentry, S. Stimpson, and J. Ritchie, "Watts Bar Unit 2 Startup Results with VERA," Oak Ridge National Laboratory, ORNL/TM-2017/194 (2017), https://doi.org/10.2172/1355891.

4. S. Stimpson, J. Powers, K. Clarno, R. Pawlowski, R. Gardner, S. Novascone, K. Gamble, and R. Williamson, "Pellet-clad Mechanical Interaction Screening Using VERA Applied to Watts Bar Unit 1, Cycles 1-3," Nuclear Engineering and Design, 327, pp. 172-186 (2018), https://doi.org/10.1016/j.nucengdes.2017.12.015.

5. T. Lange, A. Galimov, J. Eller, K. Epperson, A. Godfrey, and L. Linik, "Neutronics and CRUD Anlyses of the NuScale Small Modular Reactor," Proceedings of the Consortium for Advanced Simulation of Light Water Reactors Virtual Meeting, November 2020, pp. 198-203 (2020).

6. A. Gerlach and B. Kochunas, "Validation of RIA with SPERT," Consortium for Advanced Simulation of Light Water Reactors Technical Report CASL-U-2018-1643 (2018).

7. V. Kucukboyaci, B. Kochunas, T. Downar, A. Wysocki, and R. Salko, "Evaluation of VERA-CS Transient Capability for Analyzing the AP1000® Reactor Control Rod Ejection Accident," Proceedings of Physor (2018). 
8. S. Simunovic, VERAIn, Computer Software, https://www.osti.gov//servlets/purl/1232330, Vers. 00, USDOE (2015).

9. R. Salko, et al., "CTF Theory Manual," Oak Ridge National Laboratory Technical Report CASL-U2019-1886-002 (2020).

10. A. Wysocki, R. Salko, and B. Collins, "Coupling of CTF and TRACE for Modeling of Transients", ORNL/TM-2021/2077, Oak Ridge National Laboratory, 2021.

11. A. Wysocki, K. Borowiec, and R. Salko. Coupling interface to systems code for transient analysisl3:phi.trn.p19.01. Technical Report CASL-U-2019-1909-000, Consortium for Advanced Simulation of Light Water Reactors, 2019.

12. B. Collins, S. Stimpson, B. Kelley, M. Young, B. Kochunas, A. Graham, E. Larsen, T. Downar, and A. Godfrey, "Stability and Accuracy of Three-Dimensional Neutron Transport Simulations Using the 2D/1D Method in MPACT," Journal of Computational Physics, 326, pp. 612-628 (2016), https://doi.org/10.1016/j.jcp.2016.08.022.

13. B. Kochunas, et al., "VERA Core Simulator Methodology for PWR Cycle Depletion," Nuclear Science and Engineering, 185(1), pp. 217-231 (2017), https://doi.org/10.13182/NSE16-39. 
APPENDIX A. OLD VERA INPUT FOR ROD EJECTION 


\section{APPENDIX A. OLD VERA INPUT FOR ROD EJECTION}

The following is the full [STATE] and [MPACT] blocks for the rod ejection mentioned in a previous section. The rod is ejected at a constant speed over a period of $1 \mathrm{~s}$, followed by $4 \mathrm{~s}$ of power and TH evolution (with no additional changes to any [STATE] variables). Timestep sizes are 0.0025 from 0.0 to 0.5 and 0.001 from 0.5 to 1.0 , with one [STATE] block for each of those timesteps. No [STATE] blocks are required for the timesteps from 1.0 to 5.0, which vary in size.

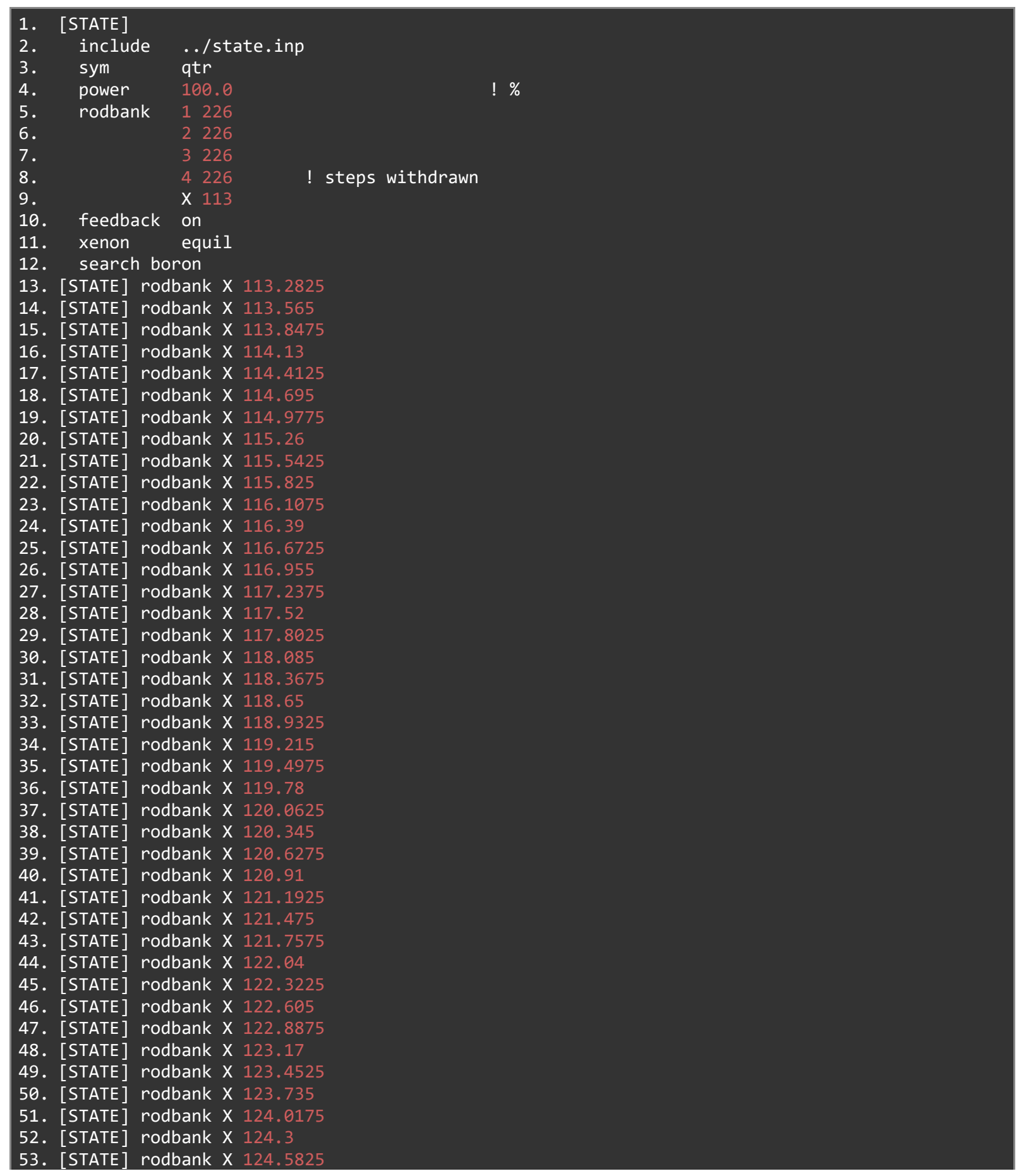




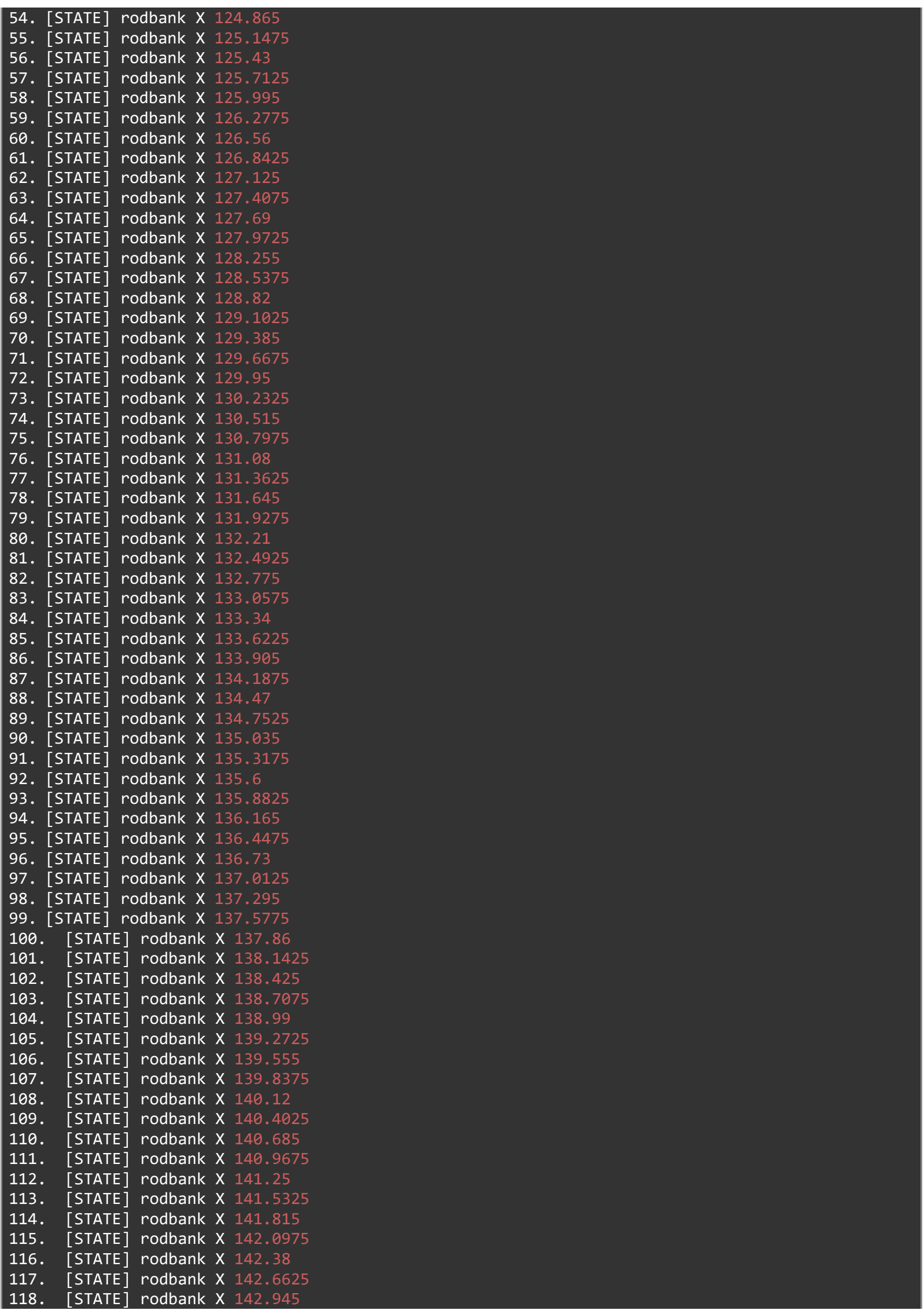




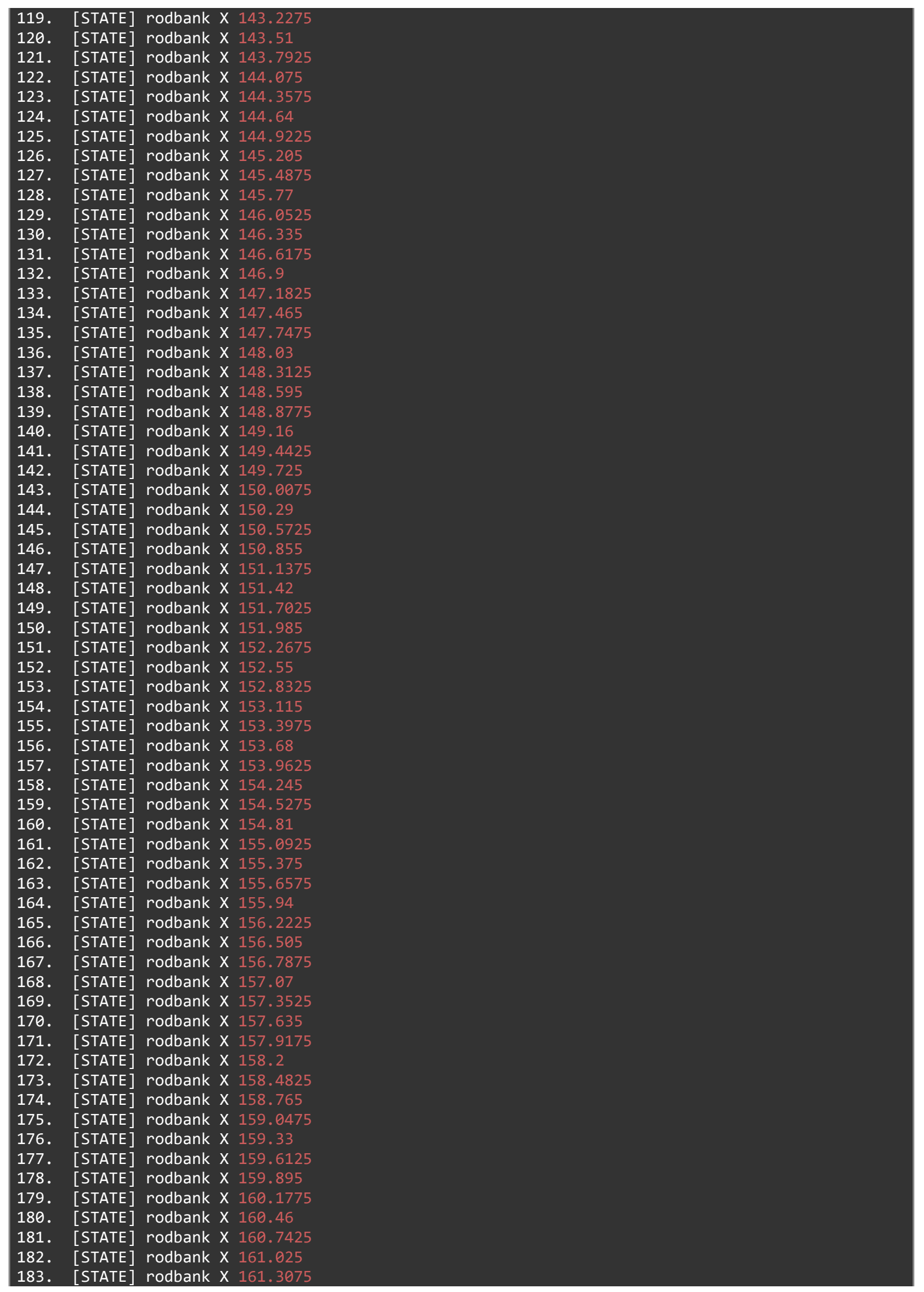




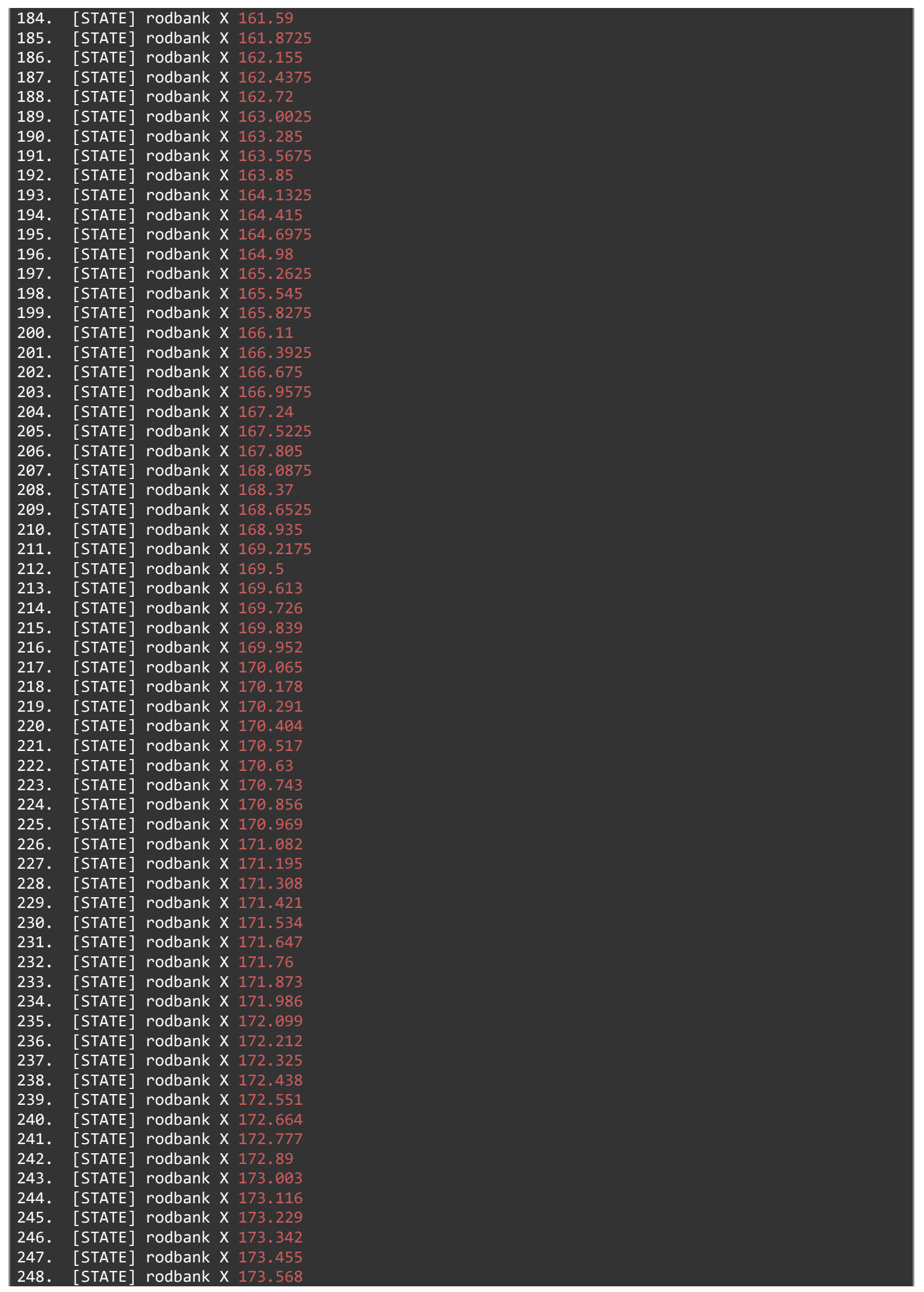




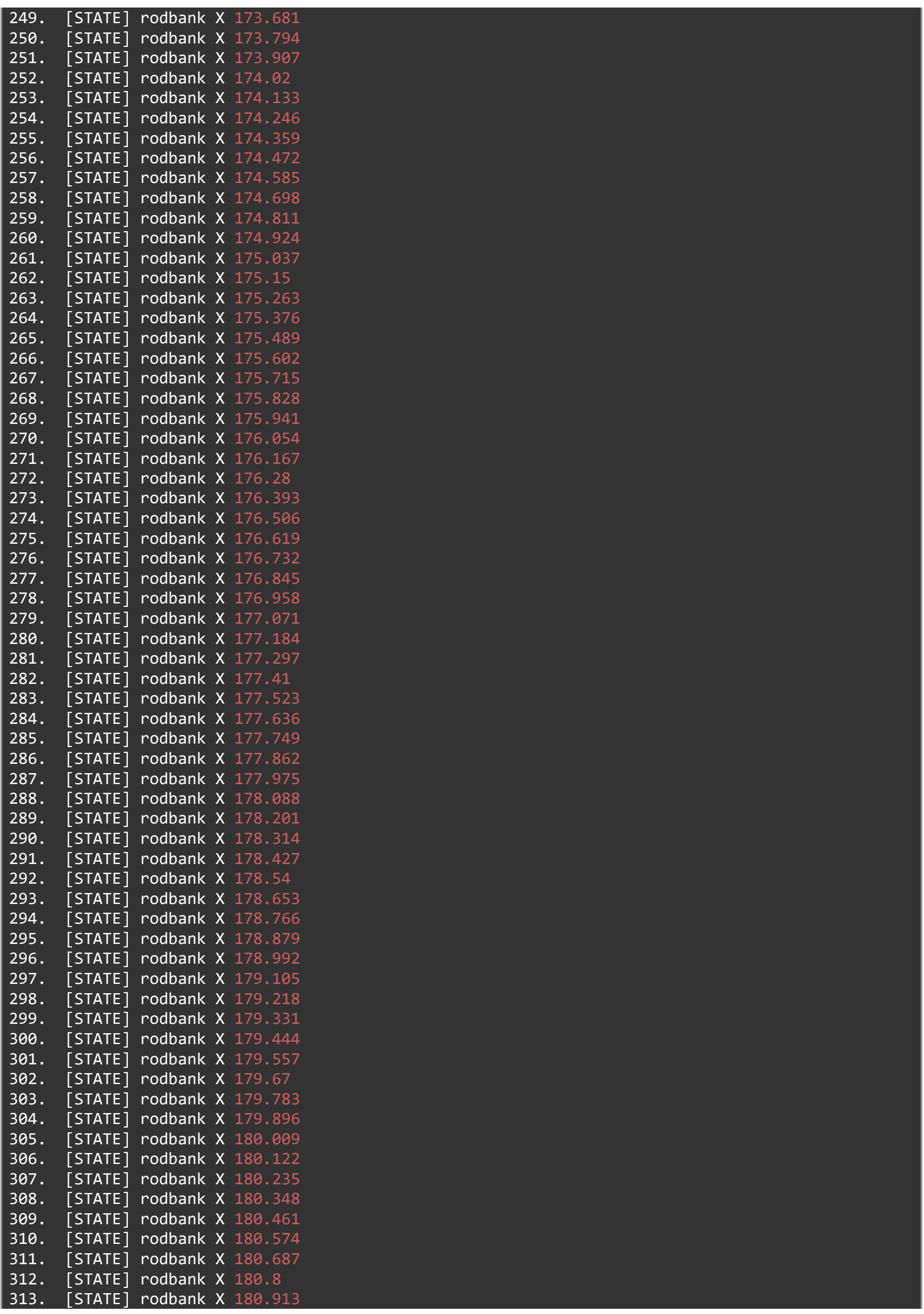




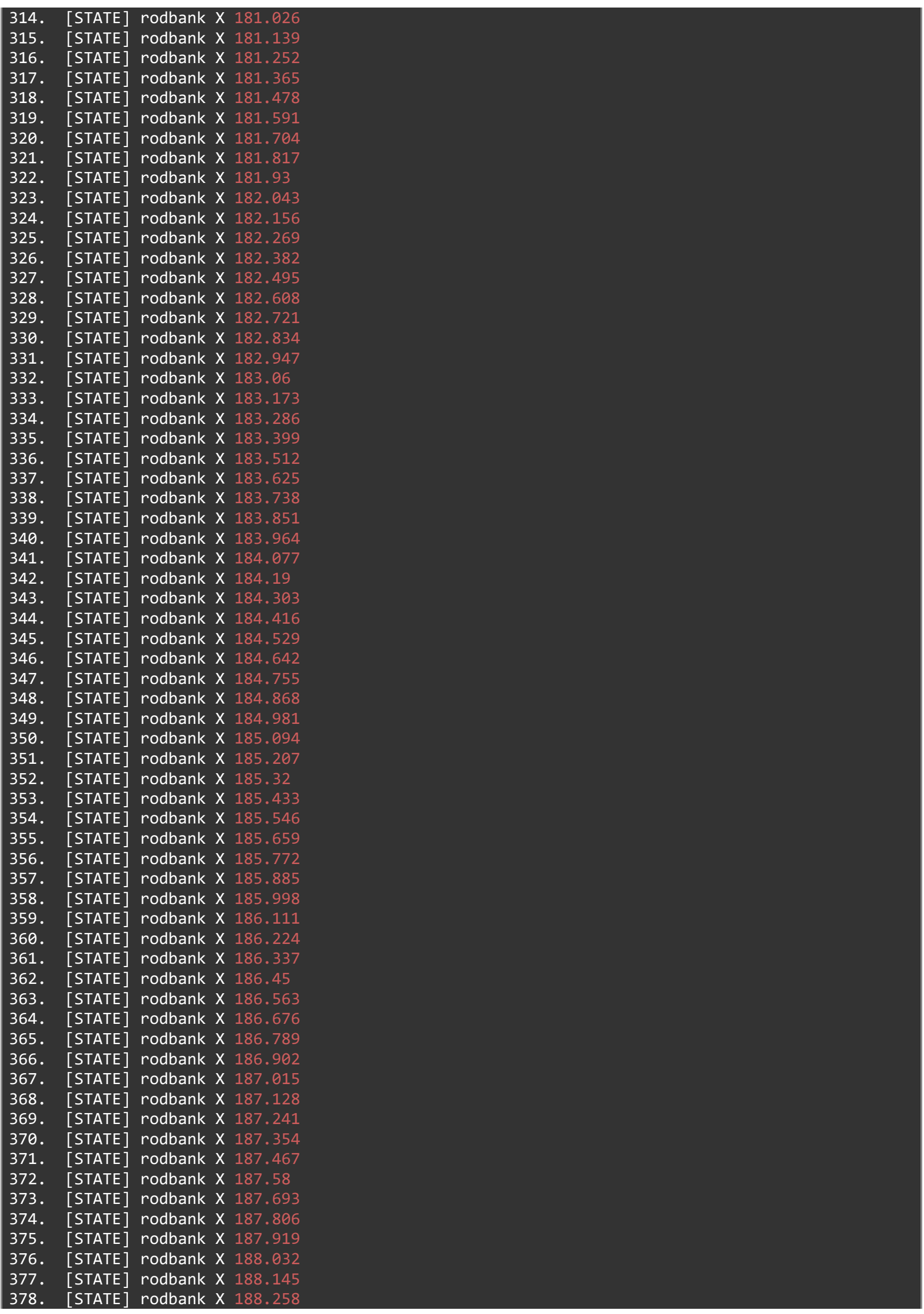




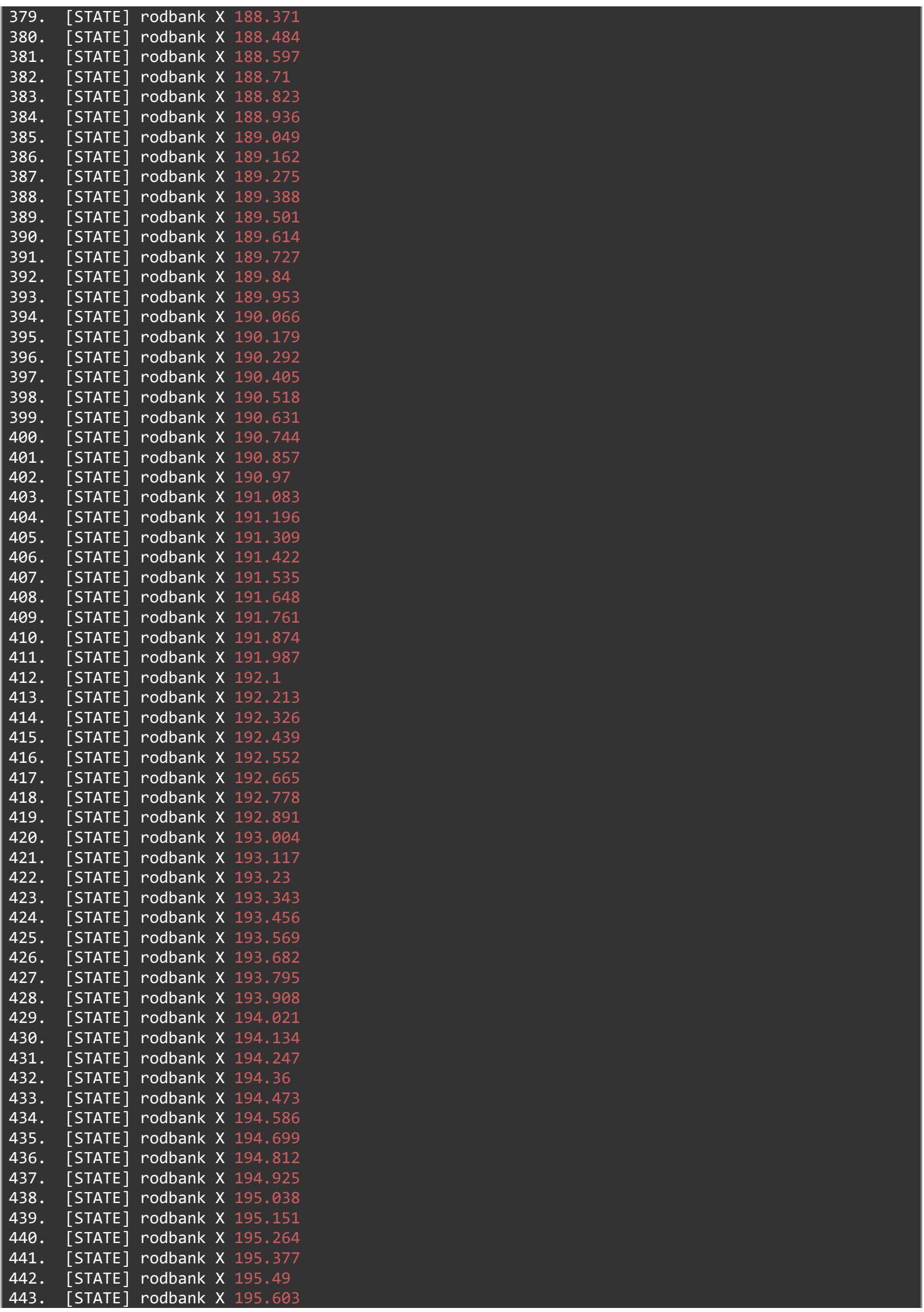




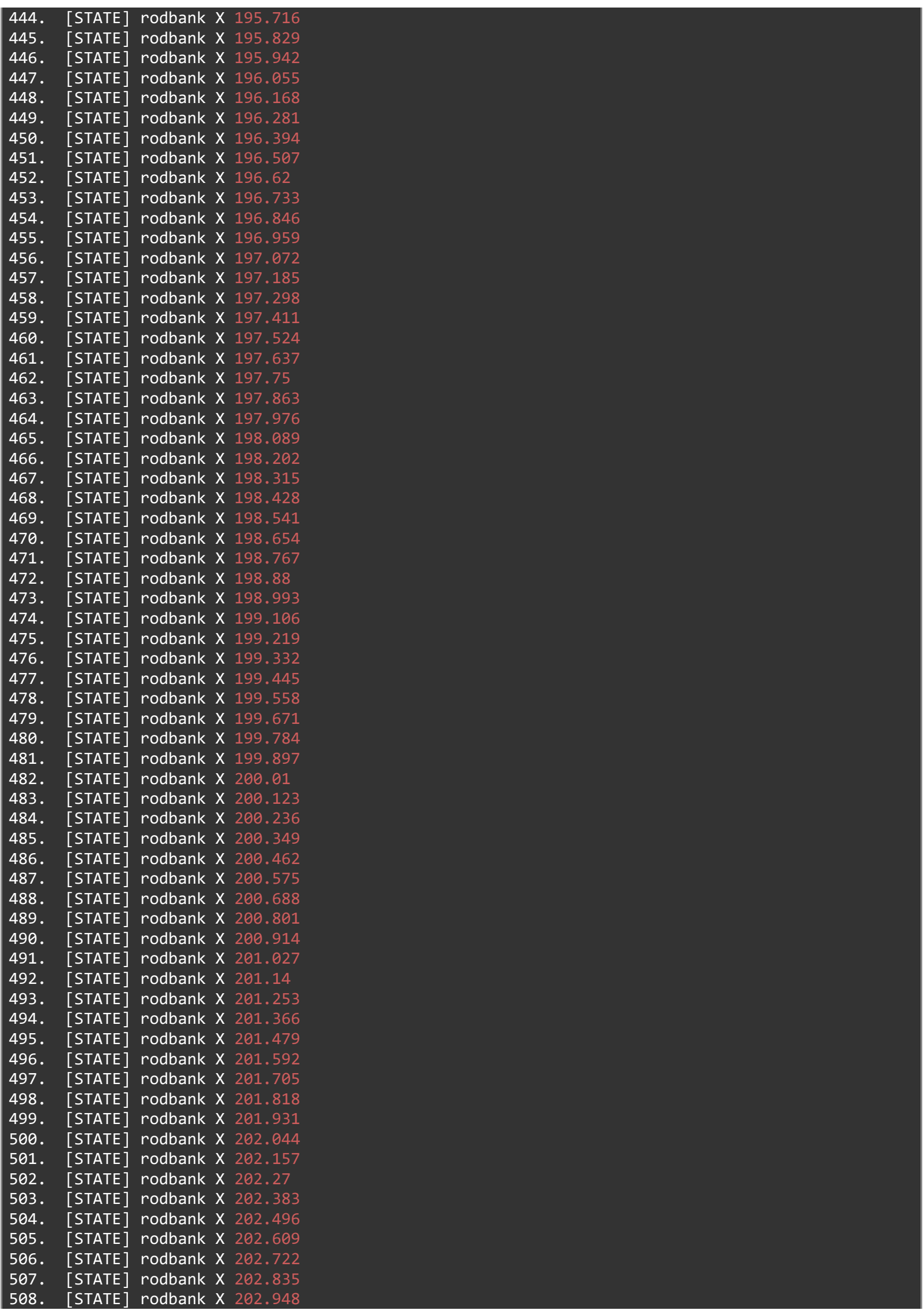




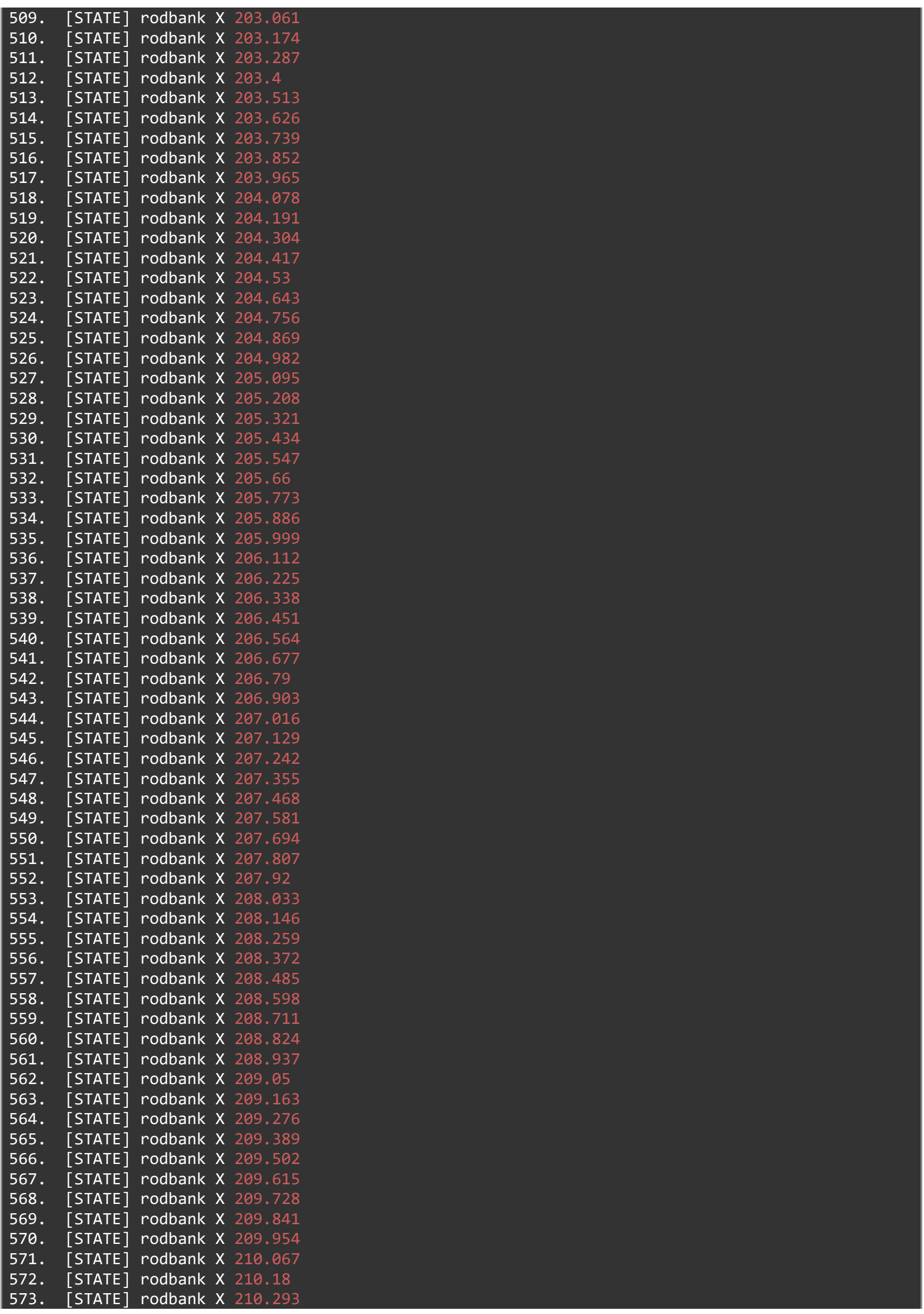




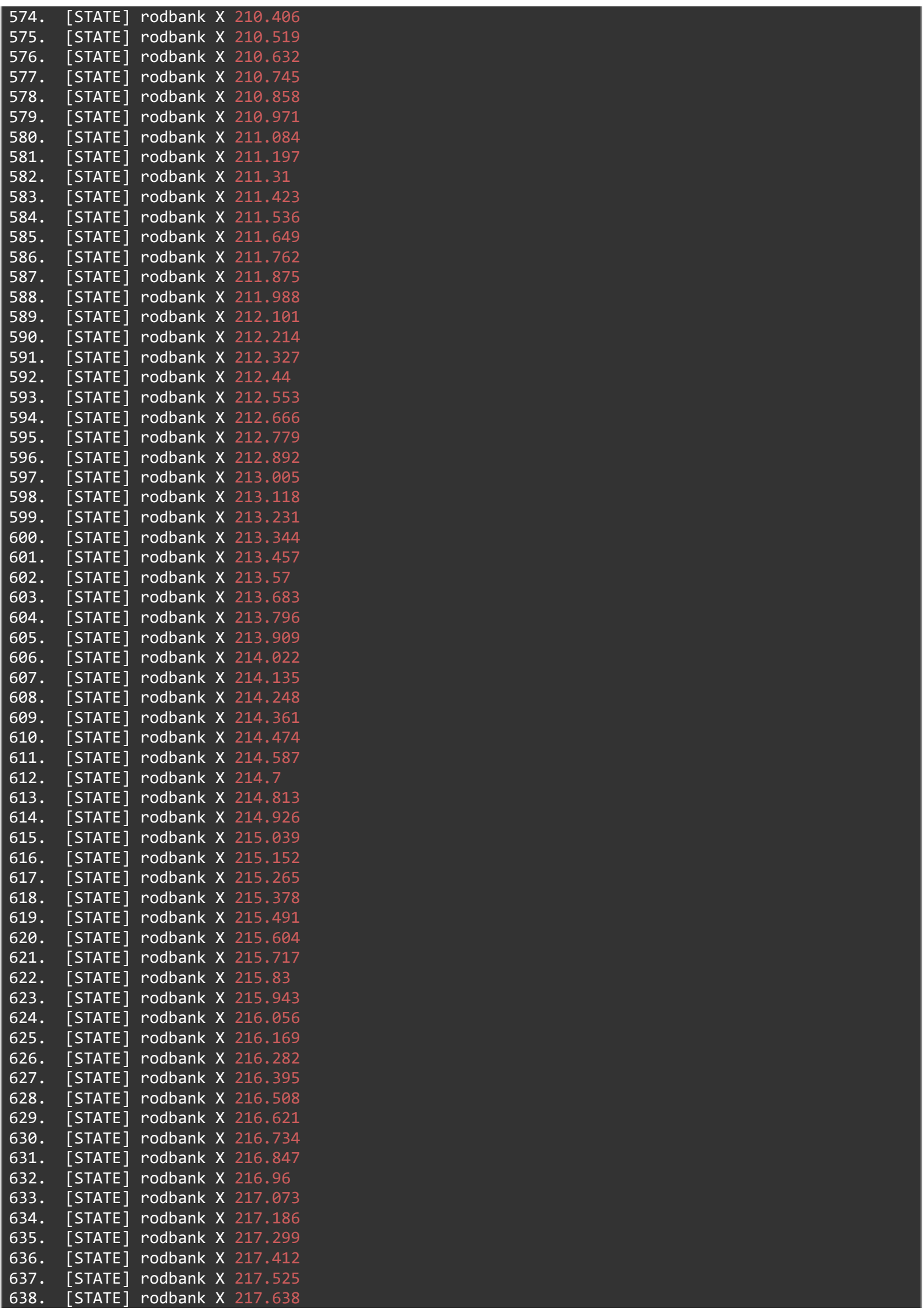




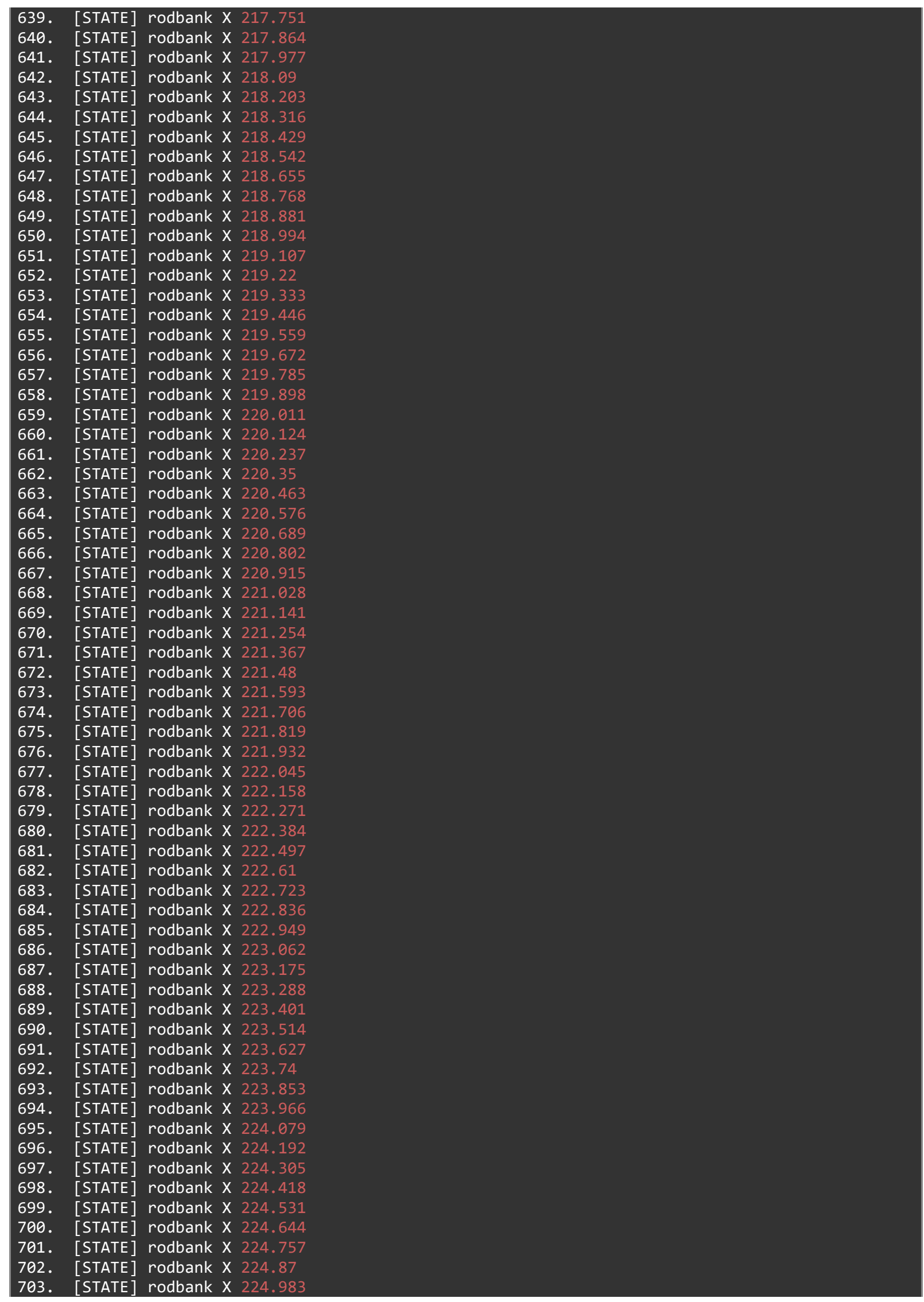


704. [STATE] rodbank X 225.096

705. [STATE] rodbank X 225.209

706. [STATE] rodbank $X 225.322$

707. [STATE] rodbank X 225.435

708. [STATE] rodbank $X 225.548$

709. [STATE] rodbank X 225.661

710. [STATE] rodbank X 225.774

711. [STATE] rodbank $X 225.887$

712. [STATE] rodbank $X 226$

713.

714. [MPACT]

715. delayenergy true

716. transmethod theta 0.5

717. prompt true

718. accel true

719. timestep 0.00250 .00255 .0

720.

721.

722.

723.

perturb $0.0 \quad 0.5 \quad 0.0025 \quad$ stcg 111

$0.51 .00 .001 \quad$ stcg 111

$\begin{array}{llllll}1.0 & 2.0 & 0.0025 \text { const } 1 & 1 & 1\end{array}$

$\begin{array}{lllllllll}2.0 & 3.0 & 0.005 & \text { const } 1 & 1 & 1\end{array}$

$\begin{array}{lllllllll}3.0 & 4.0 & 0.01 & \text { const } 1 & 1 & 1\end{array}$

725. $4.0 \quad 5.00 .025$ const 111

726 . 
APPENDIX B. OLD VERA INPUT FOR LOSS OF FLOW 


\section{APPENDIX B. OLD VERA INPUT FOR LOSS OF FLOW}

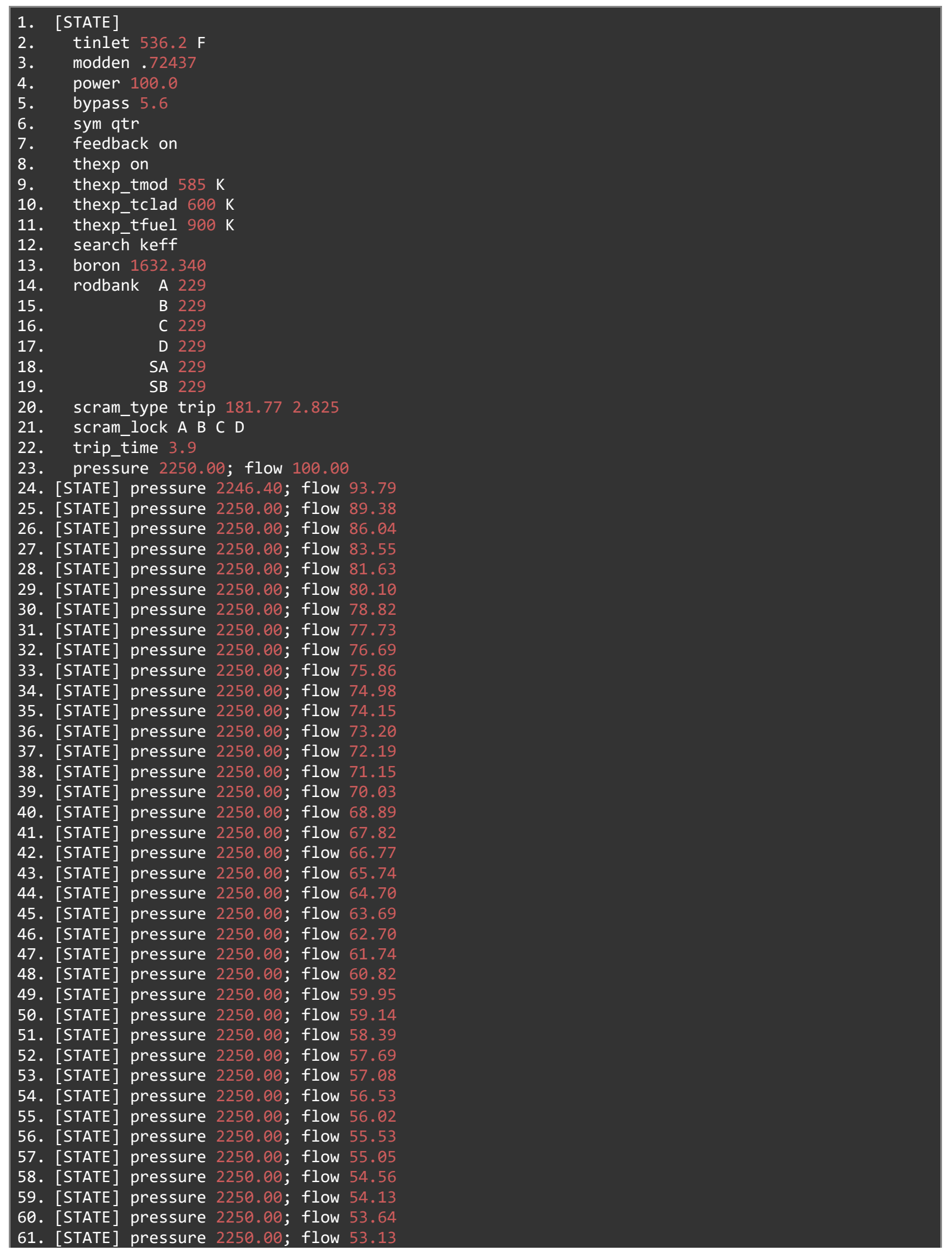




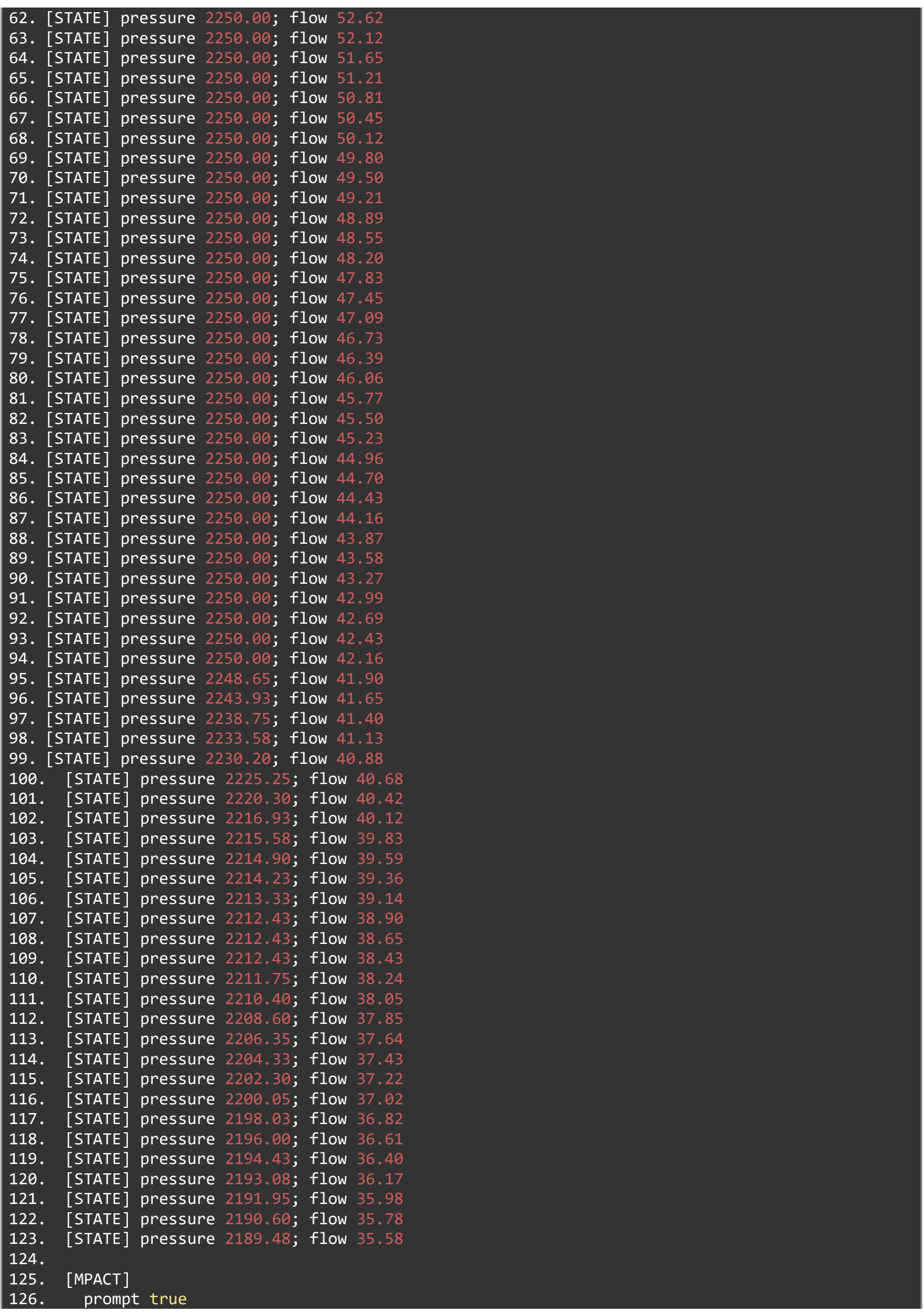


127. accel true

128. transmethod theta 1.0

129. timestep 0.100 .000110 .0

130. perturb $0.0 \quad 10.0 \quad 0.1$ stcg 112

131. 
APPENDIX C. NEW VERA INPUT FOR EXACT LOSS OF FLOW 


\section{APPENDIX C. NEW VERA INPUT FOR EXACT LOSS OF FLOW}

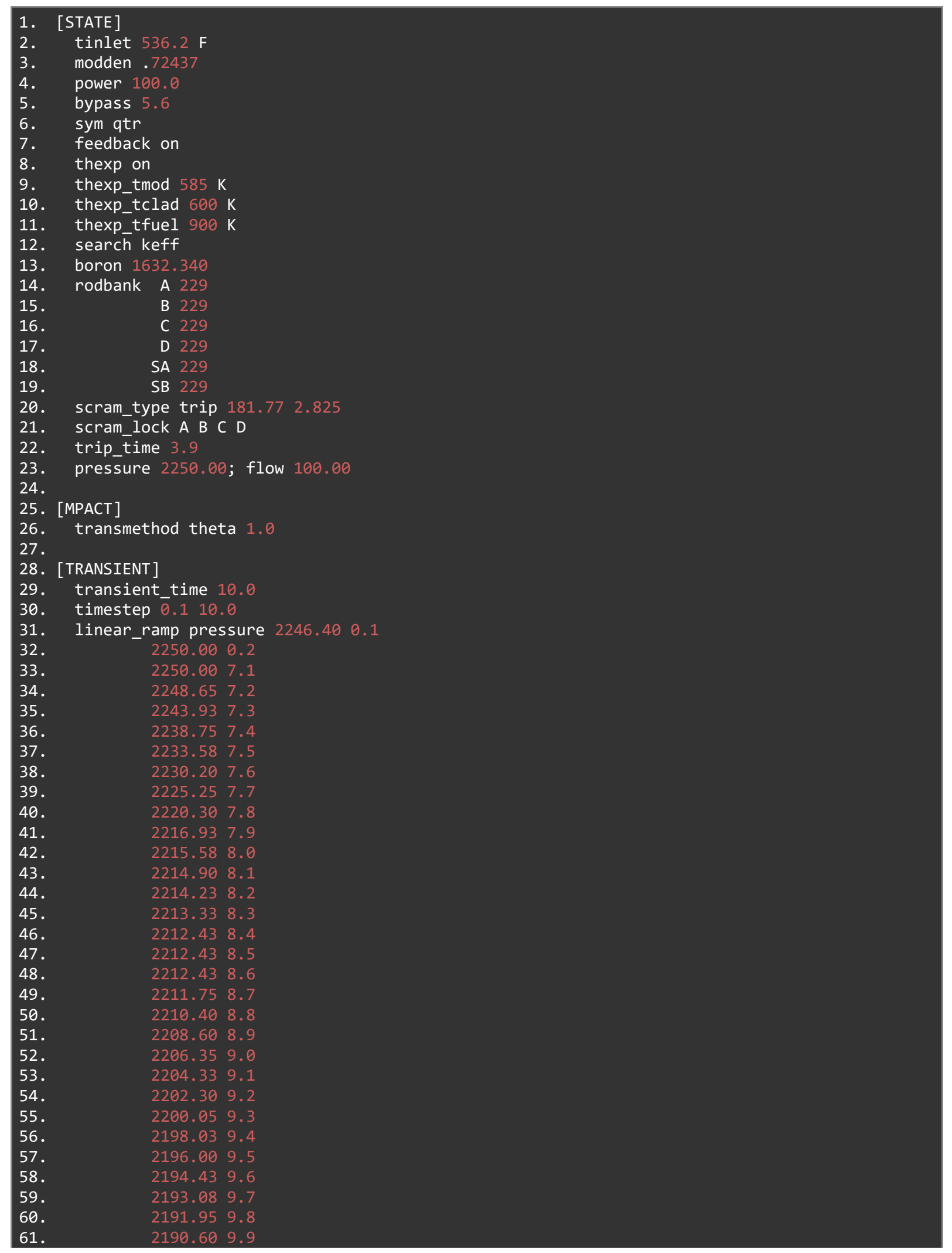




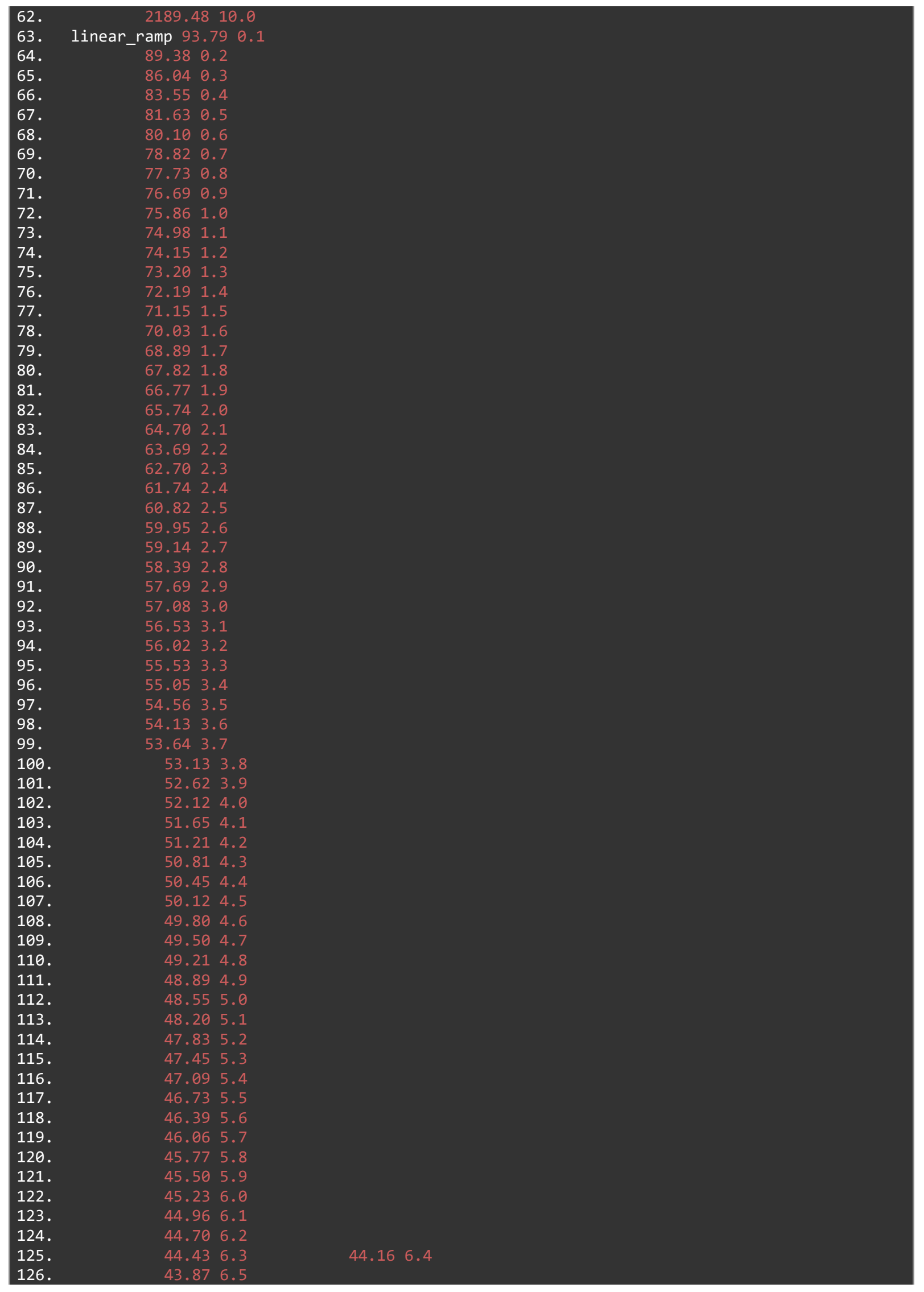




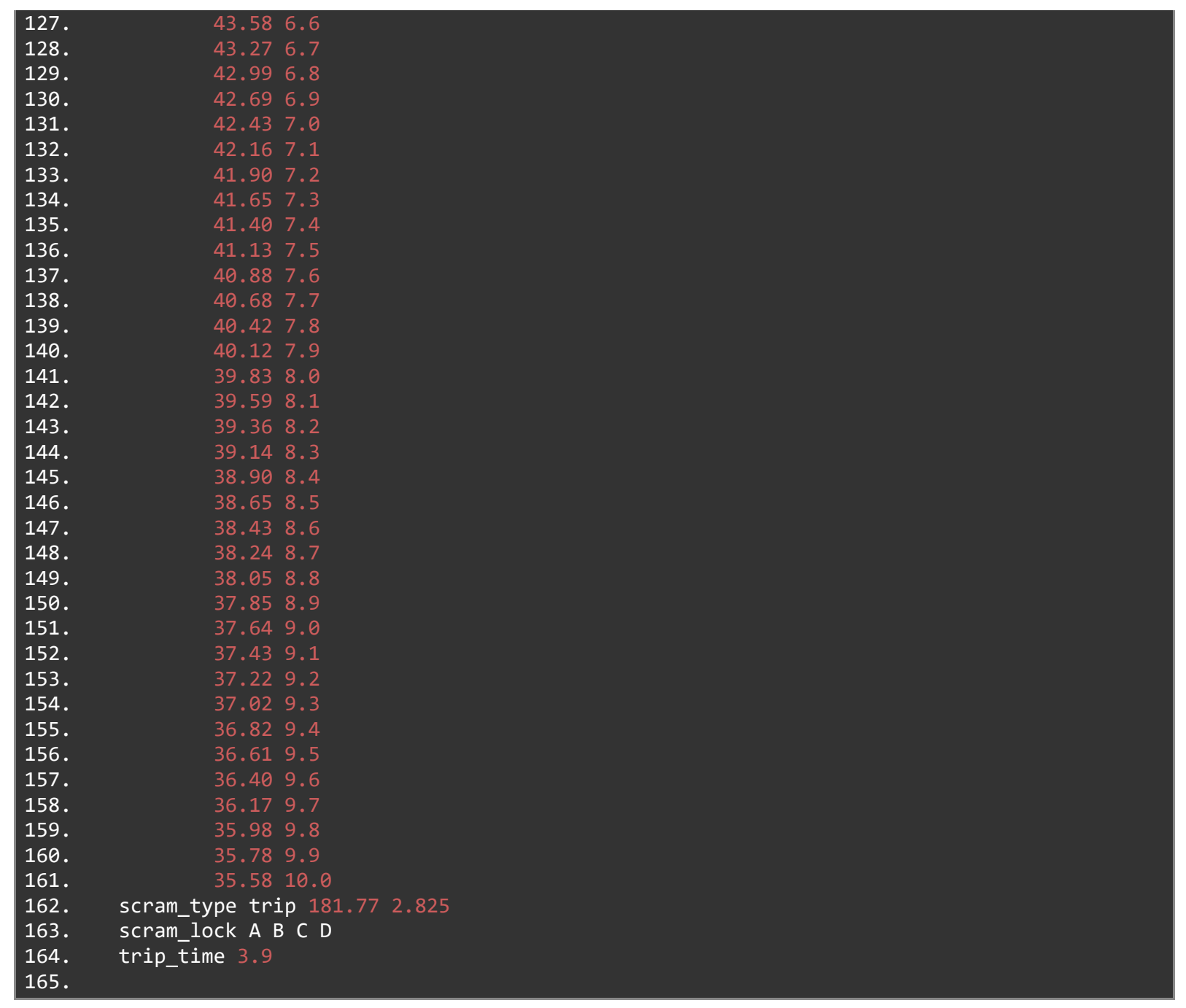

